\title{
1 Correcting index databases improves metagenomic studies
}

2

\author{
Guillaume Méric ${ }^{1,2,3 * \S}$, Ryan R. Wick ${ }^{2} *$, Stephen C. Watts ${ }^{2}$, Kathryn E. Holt ${ }^{2,4}$, Michael Inouye ${ }^{1,5,6,7}$ \\ ${ }^{1}$ Cambridge Baker Systems Genomics Initiative, Baker Heart and Diabetes Institute, 75 Commercial Rd, \\ Melbourne 3004, Victoria, Australia \\ ${ }^{2}$ Department of Infectious Diseases, Central Clinical School, Monash University, Melbourne, Victoria 3004, \\ Australia \\ ${ }^{3}$ The Milner Centre for Evolution, University of Bath, Claverton Down, Bath, BA2 7AY, UK \\ ${ }^{4}$ London School of Hygiene \& Tropical Medicine, London WC1E 7HT, UK \\ ${ }^{5}$ Cambridge Baker Systems Genomics Initiative, Department of Public Health and Primary Care, University of \\ Cambridge, Cambridge CB1 8RN, UK \\ ${ }^{6}$ Cambridge Substantive Site, Health Data Research UK, Wellcome Genome Campus, Hinxton, UK \\ ${ }^{7}$ The Alan Turing Institute, London, UK \\ * Corresponding authors: guillaume.meric@ baker.edu.au; rrwick@gmail.com; \\ $\S$ These authors have contributed equally.
}

Keywords: taxonomic classification, species definitions, metagenomics, GTDB

Running title: Larger indices improve metagenomic classification

\begin{abstract}
$\underline{\text { Abstract }}$
Assessing the taxonomic composition of metagenomic samples is an important first step in understanding the biology and ecology of microbial communities in complex environments. Despite a wealth of algorithms and tools for metagenomic classification, relatively little effort has been put into the critical task of improving the quality of reference indices to which metagenomic reads are assigned. Here, we inferred the taxonomic composition of 404 publicly available metagenomes from human, marine and soil environments, using custom index databases modified according to two factors: the number of reference genomes used to build the databases, and the monophyletic strictness of species definitions. Index databases built following the NCBI taxonomic system were also compared to others using Genome Taxonomy Database (GTDB) taxonomic redefinitions. We observed a considerable increase in the rate of read classification using modified reference index databases as compared to a default NCBI RefSeq database, with up to a 4.4-, 6.4- and 2.2-fold increase in classified reads per sample for human, marine and soil metagenomes, respectively. Importantly, targeted correction for 70 common human pathogens and bacterial genera in the index database increased their specific detection levels in human metagenomes. We also show the choice of index database can influence downstream diversity and distance estimates for microbiome data. Overall, the study shows a large amount of accessible information in metagenomes remains unexploited using current methods, and that the same data analysed using different index databases could potentially lead to different conclusions. These results have implications for the power and design of individual microbiome studies, and for comparison and meta-analysis of microbiome datasets.
\end{abstract}




\section{Introduction}

For more than 3 billion years, microbes have established complex ecological niches in environments and hosts throughout the planet. This makes them ubiquitous components of biogeochemical cycles on land [1], in the sea [2], the atmosphere [3], and on or inside other living organisms [4, 5] including humans, in which they are important for development and health [6, 7]. However, technical constraints limit our ability to study the ecology of microorganisms, in particular the widespread lack of suitable culturing methods [8]. An important advance in the analysis of microbial communities has been the use of sequence-based, culture-independent methods to study the diversity and composition of clinical and environmental samples and their biological functions. The increasing affordability of high-throughput sequencing has led to an increase in metagenomics studies, in which a sample's total extracted DNA can be sequenced as a whole. Accurately determining and quantifying the taxonomic composition of a metagenome is a critical first step in many analyses, such as the association with host phenotype, host genotype, disease status or environmental properties.

Metagenomic classification begins with the accurate assignment of sequencing reads to a reference database, or "index", comprising reference genomes and their corresponding taxonomic definitions. A wealth of metagenomic classification algorithms have been developed in the last few years [4, 9-16], mainly focusing on improving classification speed and memory usage, including popular methods such as Kraken [17, 18] or Centrifuge [19]. A given read sequence may be shared among closelyrelated species, particularly when the read length is short, and so classifiers can assign reads to the last common ancestor (LCA) of all taxa sharing their sequence ("LCA-classification"). Despite the development of ever-more efficient classifying algorithms and tools, comparatively little has been done to improve the quality of the reference indices used to define the taxa to which reads are assigned. Recent efforts showed that the addition of new genomes to NCBI RefSeq could influence metagenomic classification performance, with indices built on most recent releases of NCBI RefSeq able to classify more reads overall, but fewer at the species level [20]. Generally, most methods and studies use a selection of representative, often complete genomes from curated repositories to build indices from all described bacteria and archaea using their reported taxonomic definitions [16, 21], typically NCBI RefSeq [22] for whole representative genomes, and SILVA, Greengenes, or RDP [23] for 16S rRNA-based studies.

Defining accurate monophyletic bacterial species boundaries has always been a challenge. Bacterial taxonomy has historically been defined using imprecise biochemical or ecological phenotypes, with more recent genotyping studies offering numerous examples of clustered "species" previously thought to be distinct, and vice versa [24-27]. As a result, microbial taxonomies in reference repositories are riddled with inconsistencies, with described taxa often forming polyphyletic groupings [28, 29], necessitating reconciliation between microbial systematics and genomics [30]. This has recently been addressed by redefining taxonomic definitions using a phylogenetic depth coefficient inferred from a robust prokaryotic phylogeny [28]. This effort, summarised in the Genome Taxonomy Database (GTDB), aims to define strictly monophyletic species groups of equivalent phylogenetic depth. It produced a wealth of novel definitions at various taxonomic levels of the microbial tree of life, redefining approximately 58\% of all previous NCBI-based taxonomic definitions [28].

Most classification tools will recommend the use of default indices, built using a set of complete genomes from NCBI RefSeq. In this study, we assessed the potential for improvement and addressed the following questions: does the choice of reference index affect the performance of metagenomic classification? Does the addition of draft reference genome sequences improve classification? Should we use default NCBI-based indices, custom human microbiome-enhanced indices; or GTDB-based indices? Is the inclusion of metagenome-assembled genomes (MAGs) beneficial? Is the strict monophyly of taxonomic definitions in indices important for classification performance? To answer these questions, we created seven custom indices (Table 1) using NCBI-based and GTDB-based taxonomic definitions, and examined their classification performance on samples from three diverse and representative metagenomic datasets: human body sites, marine and soil. Our work addresses the metagenomic classification bias, whereby sequencing reads for particular taxa are present in 
103 metagenomics data but remain unclassified using current methods and recommendations. This has

104

105

106

107

108

109

110

111

112

113

114

115

116

117

118

119

120

121

122

123

124

125

126

127

128

129

130

131

132

133

134

135

136

137

138

139

140

141

142

143

144

145

146

147

148

149

150

151

152

153

154

155

156

157 important consequences for the classification of metagenomic datasets and downstream applications such as microbiome-wide association studies.

\section{$\underline{\text { Results }}$}

\section{Substantial improvements in classification performance can be achieved using larger indices}

To examine the impact of custom indices on metagenomic classification performance, we classified 404 metagenomic samples from three different datasets using seven custom indices (Figure S1, Table 1) and quantified the proportion of reads per sample that were classified to any taxon and the proportion that remained unclassified (Figure 1, Figure S2). Our custom index databases were corrected for two distinct factors: (a) number of reference genomes for each species used to build the index, and (b) strict monophyletic species definition for these reference genomes (Table 1). We observed a drastic improvement in classification performance using custom indices, built with more reference genomes, i.e. the greater the number of reference genomes used to build the index, the greater the proportion of reads classified (Figure 1A-C). This effect was not associated with sequencing depth (Figure S3). For instance, using the NCBI_r88_Human17k index on human metagenomes, which includes only 1.67-fold more genomes than NCBI_r88 (selectively chosen from 70 known human microbiome taxa) and monophyly correction (Table 1), the median proportion of classified reads per sample increased from $54.7 \%$ to $76.5 \%$. The index built with the largest number of genomes, GTDB_r86_46k, consistently classified the most reads in every sample tested. The increase in the median percentage of classified reads per sample from a default NCBI_r86 classification for human metagenomes was from $53.6 \%$ to $91.3 \%$ (median increase of $+69.4 \%$; range of $+3.9 \%$ to $+342.8 \%$ ) (Figure S2A, Table S2, Table S3). Similarly, the increase in classified reads per sample for marine metagenomes was from a median of $14.1 \%$ to a median of $55.2 \%$ (median increase of $+276.2 \%$; range of $+94.6 \%$ to $+536.3 \%$ ); and in soil metagenomes from $33.2 \%$ to $66.3 \%$ (median increase of $+100.7 \%$ reads/sample; range of $+85.7 \%$ to $+120.6 \%$ ) (Figure S2B-C, Table S2, Table S3).

We next show that the number of reference genomes rather than strict monophyly of the index database led to increased classification rate. To do so, we compared two indices built using the same reference genomes with (GTDB_r86_8.6k) and without (NCBI_r86) strict monophyletic definitions. When considering all three datasets together, the proportion of unclassified and classified reads/sample were nearly identical using GTDB_r86_8.6k over NCBI_r86 (median increase of +71 classified reads per sample, range of difference from 0 to $+2,213$ reads/sample, equivalent to a median increase of less than $+0.0005 \%$ of total reads/sample) (Figure 1, Table S2, Table S3), indicating that strict monophyly alone does not substantially affect classification rate. On the other hand, the comparison of classification performance using GTDB_r86 vs GTDB_r86_46k captures the effect of adding reference genomes ( 28,560 vs 46,006 total genomes, respectively) to two similarly monophyletic indices. When compared to GTDB_r86, GTDB_r86_46k produced more classified reads in almost every (402/404) human and environmental sample tested (Figure 1, Figure S2, Table S2, Table S3), with median percentage change in classified reads/sample of $+2.7 \%$ in human samples (range of $-5.6 \%$ to $+18.7 \%$ ), $+3.2 \%$ reads/sample in marine metagenomes (range of $+1.8 \%$ to $+5.0 \%$ ) and $+2.1 \%$ (range of $+1.9 \%$ to $+2.3 \%$ ) in soil metagenomes.

In human samples, a median of $8.6 \%$ of total reads/sample (range of $0.9 \%$ to $31.6 \%$ ) (Table S2) remained unclassified even when using our best corrected index GTDB_r86_46k. To investigate what these remaining unclassified reads are, we reclassified them using a pre-computed index based on the nucleotide (nt) database of NCBI, which excludes any whole genome sequence from the WGS or RefSeq databases but includes sequences from all taxonomic domains of life (results in Figure S4). The large majority of these reads remained unclassified ( $~ 8.5 \%$ of total reads/sample); a substantial proportion were attributed to eukaryotic $(\sim 0.86 \%$ of total reads/sample) and viral $(\sim 0.16 \%$ of total reads/sample) taxa, which are not included in the custom indices used in this study (Figure S4). Notably, $\sim 1.1 \%$ of all reads/sample were still attributed to Bacteria and Archaea (Figure S4). As the nt database of NCBI excludes reference genomes from WGS and RefSeq, this classification reflects 
158 either the presence of genomic fragments in the nt database that are not in WGS of RefSeq, or that 
213 (Figure S5B). In human samples, up to 20\% of all reads that remained unclassified using NCBI_r88 rest being attributed to a variety of other genera (Figure S5C, S5D). When examining particular species of interest, the detection of Lactobacillus crispatus in vaginal samples, Haemophilus parainfluenzae, Campylobacter concisus and Campylobacter showae in buccal and throat samples, were particularly improved by the use of the corrected NCBI_r88_Human17k index, along with numerous distinct species of Prevotella, Bacteroides and Alistipes in samples from various body sites (Figure S5C, S5D). Our results demonstrate that increasing the number of reference genomes for specific genera of interest can substantially improve their detection levels.

\section{Impact of metagenome-assembled genomes on classification performance}

The recently published GTDB taxonomic system (release 86.0) includes 3,087 metagenomeassembled genomes (MAGs) in the taxonomic redefinition of the prokaryotic tree of life [28]. We assessed whether the addition of these potentially new taxa to a reference index improved metagenomic classification on the human, marine and soil test datasets. The addition of 3,087 MAGs to GTDB increased the proportion of reads classified by mean $+0.72 \%$ (human), $+0.63 \%$ (marine) and $+0.51 \%$ (soil) (GTDB_r86_noMAGs vs GTDB_r86 index; Figure 1, Figure S2, Table S2-S3). These results show that adding MAGs to index databases can in principle increase classification performance. However, this increase was limited in our test, likely because the MAGs included in GTDB release 86.0 do not capture many novel sequences from the microbiomes analysed (human, marine, and soil).

GTDB-based species definitions affect taxonomic composition, abundance and diversity metrics, downstream analyses and interpretation from metagenomic studies

The use of corrected indices had a substantial effect on downstream metagenomic analyses. We compared the 30 most abundant taxa for HMP samples at the family, genus and species levels, from classifications using NCBI_r88, NCBI_r88_Human17k and GTDB_r86_46k (Figure 2, Figure S6). A total of $19(63 \%)$ families, $15(50 \%)$ genera and $7(23 \%)$ species appeared in the top 30 taxa using all three indices. Thus, the higher the taxonomic order examined, the more agreement across index databases (Figure 2A, Figure S6). Notably, even for taxa in the top 30 using all three indices, the order of abundance varied substantially (Figure 2B, Figure S6). Some of this variation was attributable to many taxa having been reclassified and renamed in the larger, monophyly-corrected databases (particularly GTDB_r86_46k, see yellow bars in Figure 2A). The increased taxonomic granularity within the GTDB system sometimes led to previously common taxa being divided and redefined as multiple different sub-lineages, each with a distinct taxon name. However, there were also differences in the relative abundances of top 30 taxa that were not explained by this (Figure S7). For example, the relative abundance rank of families Porphyromonadaceae and Corynebacteriaceae were reversed using NCBI_r88 vs. GTDB_r86_46k, as were the genera Lactobacillus and Bifidobacterium, and the species Bacteroides fragilis and Bacteroides thetaiotaomicron (Figure S7).

Alpha diversity (within-sample diversity), which has been associated with various phenotypes in different microbiomes [6, 31-33], is estimated directly from taxonomic composition data and therefore showed significant differences between indices. We compared three alpha-diversity metrics at the genus level (observed genus richness, genus evenness and Shannon index at the genus level), calculated from taxonomic composition tables summarised at the genus level based on classifications of the same test data sets but using seven different index databases (Figure 3). As expected, the large GTDB-based indices showed a much higher richness, but also had an effect on the evenness of genus distribution, especially in marine metagenomes, which affected Shannon diversity index distribution (Figure 3). Notably the effect of index database on alpha diversity values varied between samples, with some increasing in value and others decreasing. In some cases these differences were substantive enough to alter the results of statistical tests for difference in alpha diversity between samples from different body sites (Figure 3B, Table S6). For example, in our subset of the HMP dataset, faecal samples were found to have significantly lower Shannon diversity than buccal samples when using the NCBI_r86 index (median of 1.44 [IQR 1.03-2.25] vs median of 2.41 [IQR 2.02-2.70] respectively, $\mathrm{p}=0.027$ ) (Table S6). A similar result was obtained using NCBI_r88 index (Table S6). However no 
268 such differences were found between faecal and buccal samples when Shannon diversity was calculated using any of the GTDB-based indices (median of 2.09 [IQR 1.62-2.83] vs median of 2.59 [IQR 2.16-2.90] respectively, $\mathrm{p}=0.999$ using GTDB_r86_8.6k) (Table S6). The situation was reversed when comparing Shannon diversity for faecal and skin samples, with significant differences obtained using GTDB_r86_8.6k (median of 2.09 [IQR 1.62-2.83] vs median of 0.81 [IQR 0.63-1.12], $\mathrm{p}=0.001$ ) but not using NCBI_r86 (median of 1.44 [IQR 1.03-2.25] vs median of 0.77 [IQR 0.631.12], $\mathrm{p}=0.965)$ (Table S6).

We also examined the effect of index database choice on beta-diversity, or between-sample diversity assessed by calculating Bray-Curtis dissimilarity between groups of samples from different sources (Figure S8, S9, S10, Table S7). The effect on beta-diversity was more subtle than for alpha diversity, with the large GTDB indices yielding greater distance estimates between groups of samples that were already dissimilar using default methods (dissimilarity above $80 \%$; Figure S8, S9), but did not significantly alter the overall clustering patterns (Figure S10).

\section{$\underline{\text { Discussion }}$}

Considerable efforts have been made to improve methods for detection of taxonomic and functional markers in complex metagenomic samples, including increasing sequencing depth, optimising classification algorithms and developing more accurate de novo metagenome assembly tools. In this study, we showed that the index database is a major source of variation in classification performance and has significant ramifications for downstream analyses, which may be substantive enough to change study conclusions (e.g. alpha diversity). Commonly utilised index databases lead to suboptimal taxonomic classification, with a minority of some read sets being classified. Increasing the number of phylogenetically consistent reference genomes in an index database in either a broad or targeted manner had consistently positive effects on increasing the proportion of reads classified (sometimes several fold higher) and classification to greater taxonomic resolution. To facilitate metagenomic analyses without the need for deeper sequencing or de novo assembly, we make freely available these improved index databases (https://github.com/rrwick/Metagenomics-IndexCorrection) for two commonly-used classifiers, Centrifuge and Kraken2 and the tools to construct them as NCBI RefSeq and GTDB expand.

We found that large indices built using recently developed and largely phylogenetically-coherent taxonomic species definitions, such as GTDB [28], greatly increased the number of classified reads. Our results suggest that more coherent taxonomic definitions and accurate taxonomic boundaries, such as those proposed within GTDB, may improve statistical power and biological interpretation of subsequent results, particularly those for compositional and diversity analyses (summarised in Figure 4). This results in greater taxon granularity, i.e. smaller, more discrete clades of similar phylogenetic depth than commonly known phylogroups, which increases classification accuracy and may improve downstream applications, such as association analysis for particular traits. For example, in microbiome-wide association studies using large cohorts, a weak association with a poorly-defined lineage may be caused by a strong association with a well-defined subset of the poorly-defined lineage (Figure 4). Furthermore, at a fixed confidence level, increasing the classification rate of a metagenomic sample offers a more accurate representation of its microbial diversity and may, as we have shown, affect study conclusions. As such, the approach we propose here facilitates improved metagenomic analysis across the full spectrum of sequencing depths. In particular, our results may facilitate "shallow sequencing" metagenomics [34] by maximising the extraction of taxonomic information from samples sequenced at lower depth, thus enabling more cost-effective comparison of thousands or tens of thousands of samples in large-scale metagenomic and multi-omics studies. Lastly, our study shows the importance of consistency in index database when comparing results across studies. Differences in reference genomes and taxonomic coherence may introduce artefacts when integrating metagenomic data across studies, and therefore care should be taken when performing combined or meta-analyses. 
323

324

325

326

327

328

329

330

331

332

333

334

335

336

337

338

339

340

341

342

343

344

345

346

347

348

349

350

351

352

353

354

355

356

357

358

359

360

361

362

363

364

365

366

367

368

369

370

371

372

373

374

375

376

377

\section{$\underline{\text { Material and Methods }}$}

\section{Description of corrected index databases}

Seven different indices, ranging in size from 8674 to 46,006 complete genomes, were built to compare the effect of various factors on metagenomic classification performance (Table 1). As the focus of this study was not to compare the performance of specific metagenomic classifier tools themselves, but rather to evaluate the impact of custom indices on metagenomic classification, we picked a recently developed classifier, Centrifuge [19], on the basis of an easy-to-use index customisation pipeline, fast metagenomic classification performance and lower RAM usage than alternative tools. Centrifuge allows for the building of custom indices (via the centrifuge-build indexer), taking as input a set of sequences with taxonomic labels and a ranked taxonomic tree describing the relationships between those labels.

The NCBI_r86 and NCBI_r88 indices were built from the default collections of complete bacterial and archaeal genomes from NCBI RefSeq releases 86 ( $n=8,674$ genomes) and 88 ( $n=10,089$ genomes), respectively, using the NCBI taxonomy tree. The NCBI_r88_Human17k index was based on the NCBI_r88 index, with the addition of 6819 further reference genomes from NCBI GenBank plus manual curation of the taxonomy for 70 common human commensal and pathogenic bacterial genera (File S1). We used the Bacsort pipeline (https://github.com/rrwick/Bacsort) to manually curate the taxonomy within each of these 70 genera to enforce strict monophyly. We also built four indices using the GTDB taxonomic system (Table 1). GTDB is based on curation of $>125,000$ whole genome sequences sourced from NCBI RefSeq and metagenome-assembled genomes (MAGs); but with taxonomic labels and tree re-defined based on phylogenetic relationships inferred from the concatenation of 120 proteins and enforcing strict monophyly [28]. We built the GTDB_r86 index from the default GTDB release 86 set of 28,941 dereplicated bacterial and archaeal genomes representative of the GTDB taxonomy [28], "dereplication" being defined as the selection of reference genomes representative of phylogenetic similarity clusters [28]. In the original GTDB publication and website, two genomes were found to be "replicates" when a set of conditions were met, typically when their Mash distance was $\leq 0.05$ ( $\sim$ ANI of 95\%) [4]. The GTDB_r86_8.6k index was built using the exact same 8,674 complete reference genomes as for NCBI_r86, but using the taxonomic labels and trees assigned by GTDB. The GTDB_r86_noMAGs index was built exactly like GTDB_r86, but excluding all 3,087 metagenome-assembled genomes (MAGs) identified in GTDB release 86. Finally, the GTDB_r86_46k index was built using a lower Mash threshold for dereplication than in the default GTDB_r86 set. Specifically, this index included a total of 46,006 reference genomes (18,634 more than GTDB_r86), each representative of similarity clusters defined using a Mash [10] distance threshold of $\leq 0.005$ ( $\sim$ ANI of 99.5\%). The tax_from_gtdb.py and dereplicate_assembly.py scripts are available in https://github.com/rrwick/Metagenomics-IndexCorrection with instructions.

\section{Metagenomic datasets}

We used a total of 404 publicly available metagenomes representing a variety of commonly-studied environments: human body sites, marine and soil environments (Table S1, Figure S1). Human samples were from the WGS-PP1 study of the Human Microbiome Project (HMP) and obtained through the HMPDACC.org website [9]. HMP samples were chosen with the following considerations: we kept a representative proportion of each body site represented in the WGS-PP1 study, we did not subset a body site source with less than five samples, and we excluded samples with a high ( $>90 \%$ ) proportion of low quality reads and samples with low sequencing depth. A total of 98 representative samples were selected, corresponding to $\sim 9.2 \%(n=98 / 1067)$ of the HMP WGS-PP1 study. A total of 246 marine metagenomic samples were isolated from a range of locations in epipelagic and mesopelagic waters around the world as part of the TARA Oceans survey [35, 36], and were downloaded from the EBI repository (study MGYS00002008; BioProject PRJEB1787). A total of 60 soil metagenomes were sampled in a recent study from meadows ground at various depths [37], 
and were obtained from NCBI BioProject PRJNA449266. Accessions for all readsets are listed in Table S1.

\section{Assessment of metagenomic classification performance}

For all classifications, we ran Centrifuge version 1.0.4 [19] on a Linux x86 cluster with 16 cores and $128 \mathrm{~GB}$ of RAM allocated for each sample classification. The run time ranged from 11 to 45 minutes per metagenomic sample, depending on the index used for classification and the sequencing depth of the sample. Classification reports were built from the resulting output files using the centrifugekreport tool, and reports were visualised and exported using Pavian version 0.8.1 [38] and custommade scripts, available at https://github.com/rrwick/Metagenomics-Index-Correction.

Classification performance was assessed by first comparing the number of unclassified and classified reads per sample for each index database used. This provides an unambiguous way to measure how much of the total microbial information present in each sample can be classified. We also compared the taxonomic ranks to which reads were assigned using each index. It should be noted that the NCBI prokaryotic taxonomic system includes many additional and ambiguous taxonomic ranks that are not present in GTDB, such as "subphylum", "infraclass", "superclass", "subtribe" or "strain". To make results comparable between taxonomic systems, reads were always attributed and reported to the LCA of the standard ranks: phylum, class, order, family, genus and species.

A pre-compiled index based on the nucleotide (nt) database is available from the Centrifuge website (http://www.ccb.jhu.edu/software/centrifuge/, compiled on 03/03/2018). This database includes all traditional divisions of GenBank, EMBL and DDBJ, and thus includes eukaryotic and viral sequences in addition to prokaryotes. However, the nt database excludes the WGS section of GenBank, which should have a negative impact on the determination of accurate species-specific microbial markers. Accordingly, we observed that the classification of 10 random HMP metagenomes using nt resulted in more unclassified reads than when using GTDB_r86_46k (data not shown). To investigate the origin of the reads which were unclassified by the GTDB_r86_46k index (the best-performing custom index in this study), we reclassified them using the nt database.

Finally, we assessed the effect of using different indices on commonly-used ecological diversity metrics. The calculation of alpha and beta diversity estimates (observed genus richness, genus evenness, Shannon diversity and Bray-Curtis dissimilarity at the genus level) was performed using the $\mathrm{R}$ package phyloseq version 1.24.2 [39].

\section{Custom scripts and pre-computed index databases availability}

A collection of scripts used to prepare, compare and analyse Centrifuge classifications using custom index databases, either based on default NCBI or GTDB taxonomic systems, is available at: https://github.com/rrwick/Metagenomics-Index-Correction with instructions. Pre-computed versions of the NCBI_r88_Human17k and GTDB_r86_46k indices suitable for use with Centrifuge [19], Kraken1 [18], Kraken2 (https://ccb.jhu.edu/software/kraken2/) and their variants (KrakenUniq [17], LiveKraken [40]), are freely available to from: https://monash.figshare.com/projects/Metagenomics_Index_Correction/65534. 


\section{Competing interests}

425

426

427

428

429

430

431

432

433

434

435

436

437

The authors declare that they have no competing interests.

\section{Funding}

This study was supported in part by the Victorian Government's OIS Program. SCW was supported by a studentship funded by the Australian Government Research Program. KEH was supported by a Senior Medical Research Fellowship from the Viertel Foundation of Australia, and by the Bill and Melinda Gates Foundation of Seattle.

\section{Authors' contributions}

GM designed the study, generated performed analyses, interpreted results and was the major contributor in writing the manuscript. RRW helped generating scripts and databases, interpreted results and participated in the writing of the manuscript. SCW helped to generate code and databases. $\mathrm{KEH}$ and MI were key contributors to the study design, interpretation of results and the writing of the manuscript. All authors read and approved the final manuscript. 
443

444

445

446

447

448

449

450

451

452

453

454

455

456

457

458

459

460

461

462

463

464

465

466

467

468

469

470

471

472

473

474

475

476

477

478

479

480

481

482

483

484

485

486

487

488

489

490

491

492

493

494

495

496

\section{Figure and table legends}

Table 1. Description of the seven classification indices used in this study. The release numbers correspond to NCBI RefSeq releases of genomes from which the reference genomes used to build indices were obtained.

Figure 1. Large index databases substantially improve metagenomic classification performance and accuracy, including at lower taxonomic levels. Sequencing reads from three datasets (HMP samples, $n=98$; TARA Oceans samples, $n=246$; meadow soil samples, $n=60$ ) were classified using the seven index databases presented in Table 1. Boxplots (Tukey) show the distribution of the proportion of unclassified and classified reads/samples for human samples (A), marine samples (B) and soil samples $(\mathrm{C})$ using seven indices ( $\mathrm{y}$-axis) is shown for each index size (x-axis), defined as the number of reference genomes used to build the index. Distributions of the breakdown of read classification to the two lowest taxonomic levels (genus, species) for human samples (D), marine samples (E) and soil samples (F) are shown for the NCBI_r86 default index (light blue), two indices based on NCBI_r88 (NCBI_r88 in green and NCBI_r88_Human17k in pink) and two indices based on GTDB_r86 (GTDB_r86 in orange and GTDB_r86_46k in purple).

Figure 2. Effect of index database correction on metagenomic composition. (A) Number of shared top 30 most abundant families, genera and species after classification of 98 HMP samples using default NCBI_r88 index and corrected NCBI_r88_Human17k and GTDB_r86_46k indices. (B) Comparisons of relative abundances ( $-\log _{10}$ scale) between the default NCBI_r88 classification and NCBI_r88_Human17k classifications (left) and GTDB_r86_46k (right) for taxa in the top 30 most abundant of all three classifications (19 families, 15 genera and 7 species). To assess changes in rank order consistency between the classifications, Spearman's rank correlation coefficient, and the associated p-value are shown for both comparisons of NCBI_r88_Human17k and GTDB_r86_46k classifications with NCBI_r88 for all taxa, and at each taxonomic ranks.

Figure 3. Using corrected indices to classify metagenomes affects measures of alpha diversity. (A) The values of three measures of alpha diversity (observed genus richness, genus evenness and Shannon diversity index at the genus level) for each metagenomic sample from three datasets (HMP subset, TARA Oceans, Meadow soil samples) are shown. Three specific comparisons of values are presented, between NCBI_r86 and GTDB_r86_8.6k, between NCBI_r88 and NCBI_r88_Human17k and between GTDB_r86, GTDB_r86_noMAGs and GTDB_r86_46k. Each sample is represented by a line coloured by isolation phenotype. Statistical comparisons of distributions presented in this panel are shown in Table S6. (B) Effect of classification index on alpha diversity metrics comparisons between groups. The scatter plots compare the significance of ANOVA tests on all alpha-diversity measures for each of three comparisons, $P$-values using Dunn's multiple testing (with Holm's correction). The dotted lines represent proportionality for which p-values are identical for classifications using both indices, the red lines denote the $\mathrm{p}$-value threshold of $0.05\left(-\log _{10}=1.301\right)$ for each index.

Figure 4. Increased taxonomic granularity in classification indices can improve the interpretation of microbiome-wide association studies. (A) Increased taxonomic granularity is defined here by the accurate redefinition, splitting and merging of phylogenetically-coherent strictlymonophyletic lineages, as performed using GTDB. In this example, taxon A is split into taxa A1, A2, $\mathrm{A} 3$ and $\mathrm{A} 4$, and taxon B is split into B1 and B2. (B) Example classification using two index databases, a smaller number of reference genomes with polyphyletic definitions (left) and a larger number of reference genomes with monophyletic definitions (right). (C) Example effects of index database correction on downstream analysis involving alpha-diversity metrics (left) or for microbiome-wide association studies (right).

Figure S1. Description of 404 metagenomic samples used in this study. The distribution of the number of reads/sample is shown for 98 human (A), 246 marine (B) and 60 soil (C) samples, 
497

498

499

500

501

502

503

504

505

506

507

508

509

510

511

512

513

514

515

516

517

518

519

520

521

522

523

524

525

526

527

528

529

530

531

532

533

534

535

536

537

538

539

540

541

542

543

544

545

546

547

548

549

550

551 according to various sampling information (body site for human samples, and sampling depth for marine and soil samples).

Figure S2. Per-sample change (\% and fold-change) in unclassified and classified reads/sample using seven default and corrected NCBI- and GTDB-based indices. Per-sample percent and foldchanges are shown for the three metagenomic datasets: (A) human samples ( $n=98)$, (B) TARA Oceans samples $(n=246)$ and $(C)$ meadow soil samples $(n=60)$. Values are normalised to the number of reads unclassified and classified using the default NCBI_r86 index.

Figure S3. Classification improvement using corrected indices is unaffected by variations in sequencing depth. The total number of reads/sample (a proxy for sequencing depth) was plotted against the number of unclassified reads/sample using 6 default and corrected indices, for human (A), marine (B) and soil (C) metagenomes. The regression line was calculated using a linear model fit ("lm" in ggplot2 geom_smooth function) for each index.

Figure S4. Reads from human metagenomes that remained unclassified using the GTDB_r86_46k index are mostly unknown and eukaryotic. (A) Proportion of reads/sample from HMP samples ( $\mathrm{n}=98$ ) that are unclassified using GTDB_r86_46k, according to body site of isolation; (B) and (C) outcome of re-classification of these specific reads using an index based on the NCBI nucleotide database (nt; pre-computed on the 03/03/2018 and available on the Centrifuge website [http://www.ccb.jhu.edu/software/centrifuge/]) in number of reads/sample (B) and in proportion (C); (D) per-sample breakdown of domain re-classification, showing the proportion of reads attributed to Eukaryota, Bacteria, Archaea and Viruses or unclassified.

Figure S5. Targeted correction for 70 specific bacterial genera increases their detection levels in human metagenomes. (A) The average number of reads classified to all 1001 different bacterial genera to which at least one read was attributed using the NCBI_r88 and the NCBI_r88_Human17k indices were compared, with the 70 specifically corrected genera were highlighted in blue while the non-corrected genera are shown in red. Any point above the line denotes genera to which more reads were classified using the NCBI_r88 index, while any point below the line denotes genera to which more reads were classified using the NCBI_r88_Human17k index. (B) Number of classified reads/sample using a default index and a targeted correction for 70 specific bacterial genera. The 70 corrected genera are shown, along with their corresponding distribution of the number of classified reads/sample using NCBI_r88 (red) or NCBI_r88_Human17k (blue). The column on the right indicates the p-value and significance thresholds after Wilcoxon signed-rank tests comparing the two indices. Genera with the highest significance in difference are shown in red, orange and yellow, and non-significant differences are shown in white. (C and D) From all reads that were unclassified using NCBI_r88 but classified using NCBI_r88_Human17k, the top 30 genera (C) and species (D) to which these reads were attributed, in proportion, are shown. Boxplots of different colours denote different isolation sources, showing how body sites are differently affected by the index correction.

Figure S6. Effect of index database correction on metagenomic compositional data and most abundant taxa. The 30 most relative abundant families, genera and species to which reads were attributed using three indices (default NCBI_r88 and corrected indices NCBI_r88_Human17k and GTDB_r86_46k) are shown in boxplots. The colour of the taxon name in the y-axes denotes whether the same taxon label was found to be present in the top 30 most abundant taxa after classification by all three indices (blue), by NCBI_r88 and NCBI_r88_Human17k and not GTDB_r86_46k (orange), by GTDB_r86_46k and either NCBI_r88 or NCBI_r88_Human17k (pink) or only in one index (black). In red are indicated the taxon definitions that are existing only in one index. For the comparison at the genus level, green arrows indicate whether the corresponding genus has been specifically corrected in the NCBI_r88_Human17k index.

Figure S7. Comparison of metagenomic compositional data and most abundant taxa after classification with indices built from the same reference genomes taxonomically defined using NCBI- or GTDB-based definitions. The 30 most relative abundant families (A), genera (B) and 
552 species (C) to which reads were attributed using the NCBI_r86 and GTDB_r86_8.6k indices, built with the exact same set of complete reference genomes from NCBI RefSeq release 86, are shown in boxplots. The correspondence between the top 30 most abundant taxa from the two classifications is reflected by lines between the two plots. The colouring of the lines denote taxa with an exact correspondence in both indices (plain blue) or whether the GTDB redefinition of taxa affected the correspondence (dotted red). The taxa written in red were created in GTDB.

Figure S8. Effect of using corrected indices to classify metagenomes on Bray-Curtis dissimilarity between groups of HMP samples (grouped by body site isolation). (A) Bray-Curtis dissimilarity distributions are shown for pairwise group comparisons between buccal, throat, skin, faecal and vaginal samples of the HMP dataset subset $(n=98)$ used in this study, using seven different classification indices. Coloured panels denote within-group comparisons, white panels denote between-group comparisons. (B) Visualisation of the same data, but ordered to contrast the effect of index database on pairwise group comparisons of Bray-Curtis dissimilarity. Colours represent body sites, similarly to panel A.

Figure S9. Effect of using corrected indices to classify metagenomes on Bray-Curtis dissimilarity between groups of TARA Oceans and meadow soil samples (grouped by body site isolation). (A-B) Bray-Curtis dissimilarity distributions are shown for pairwise group comparisons between buccal, throat, skin, faecal and vaginal samples of the TARA Oceans dataset ( $n=246$, panel A) and meadow soil dataset ( $n=60$, panel B) used in this study, using seven different classification indices. Coloured panels denote within-group comparisons, white panels denote between-group comparisons. (C-D) Visualisation of the same data, but ordered to contrast the effect of index database on pairwise group comparisons of Bray-Curtis dissimilarity for TARA Oceans samples (panel C) and meadow soil samples (panel D). Statistical comparisons of distributions presented in this figure are shown in Table S7.

Figure S10. Effect of using corrected indices to classify metagenomes on Bray-Curtis dissimilarity (ordination plots). Between-sample diversity was compared by calculating and ordinating Bray-Curtis distance measures for samples classified using NCBI_r88 (C, H, M), NCBI_r88_Human17k (D, I, N) and GTDB_r86_46k (E, J, O). To compare the effect of indices on beta-diversity, we performed permutational multivariate analysis of variance (PERMANOVA) on the Bray-Curtis distances to measure the association between sample information (such as isolation source for the HMP samples, or sampling depth for the marine and soil samples) and variance within the dataset, indicated in bold in panels C-E, H-J, M-O.

Table S1. Sample description and accession number for 404 public human, marine and soil metagenomes used in this study.

Table S2. Summary of classification outcome for three datasets using seven different index databases. The median, average, minimum and maximum values of the number and proportion of classified and unclassified reads/sample is shown for every condition. Classifications were performed using Centrifuge version 1.0.4. Detailed per-sample values are shown in Table S3.

Table S3. Detailed per-sample classification outcome using samples from three datasets, after classification using seven different index databases. The number and proportion of classified and unclassified reads/sample is shown for every condition. Classifications were performed using Centrifuge version 1.0.4. A summary of these results is shown in Table S2.

Table S4. Summary of classification outcome at the genus and species level for samples from three datasets using five different index databases. The median, average, minimum and maximum values of the number and proportion of classified reads/sample is shown for every condition. Classifications were performed using Centrifuge version 1.0.4. Detailed per-sample values are shown in Table S5. 
607 Table S5. Detailed per-sample classification outcome to genus and species levels using samples 608 from three datasets, after classification using five different index databases. The number and 609 proportion of classified reads/sample is shown for every condition. Classifications were performed 610 using Centrifuge version 1.0.4. A summary of these results is shown in Table S4.

611

Table S6. Influence of index correction on the significance of differences between alpha diversity of isolation phenotype groups. Comparison of significance ( $p$-values) from Dunn's multiple testings with Holm correction after ANOVA on three alpha diversity metrics comparisons (observed richness, evenness and Shannon diversity index) between isolation phenotype groups in three metagenomic datasets (body site for HMP samples, depth of sampling for TARA Oceans and meadow soil samples) after classification with six index databases. Specifically, the alpha diversity metric distribution between isolation group pairs were compared after classifications with NCBI_r86 and GTDB_r86_8.6k, NCBI_r88 and NCBI_r88_Human17k and GTDB_r86, GTDB_r86_noMAGs and GTDB_r86_46k.

Table S7. Influence of index correction on the significance of differences between beta diversity (Bray-Curtis dissimilarity) calculated between isolation phenotype groups. Comparison of significance (p-values) from Dunn's multiple testings with Holm correction after ANOVA on Bray Curtis dissimilarity comparisons between isolation phenotype groups in three metagenomic datasets (body site for HMP samples, depth of sampling for TARA Oceans and meadow soil samples) after classification with four index databases. Specifically, comparisons were between NCBI_r88 and NCBI_r88_Human17k and GTDB_r86 and GTDB_r86_46k.

File S1. Description of the NCBI_r88_Human17k index database creation. Pre- and postcorrection Newick and XML phylogenetic trees built on hybrid FastANI/Mash distances for 9928 genomes from 70 genera of interest (Acinetobacter, Alistipes, Anaerostipes, Atlantibacter, Bacteroides, Barnesiella, Bifidobacterium, Blautia, Brenneria, Buttiauxella, Butyrivibrio, Campylobacter, Cedecea, Citrobacter, Clostridium, Coprococcus, Dickeya, Dorea, Edwardsiella, Enterobacter, Erwinia, Escherichia, Eubacterium, Faecalibacterium, Haemophilus, Hafnia, Helicobacter, Intestinimonas, Izhakiella, Klebsiella, Kluyvera, Kosakonia, Lachnoclostridium, 638 Lactobacillus, Leclercia, Lelliottia, Mangrovibacter, Moraxella, Morganella, Nissabacter. 639 Phascolarctobacterium, Phytobacter, Plesiomonas, Prevotella, Proteus, Providencia, 640 Pseudescherichia, Pseudocitrobacter, Pseudomonas, Psychrobacter, Rahnella, Raoultella, Roseburia, 641 Rosenbergiella, Rouxiella, Ruminiclostridium, Ruminococcus, Salmonella, Serratia, Siccibacter, 642 Tatumella, Trabulsiella, Xenorhabdus and Yersinia), suitable for visualisation in Archeopteryx [41]. 643 The species definitions for these genomes were corrected and made strictly monophyletic to create the 644 "NCBI_r88_Human17k" index. 
647

648

649

650

651

652

653

654

655

656

657

658

659

660

661

662

663

664

665

666

667

668

669

670

671

672

673

674

675

676

677

678

679

680

681

682

683

684

685

686

687

688

689

690

691

692

693

694

695

696

697

698

699

700

701

\section{$\underline{\text { References }}$}

1. van der Heijden MG, Bardgett RD, van Straalen NM: The unseen majority: soil microbes as drivers of plant diversity and productivity in terrestrial ecosystems. Ecol Lett 2008, 11:296-310.

2. $\quad$ Stocker R: Marine microbes see a sea of gradients. Science 2012, 338:628-633.

3. Vaitilingom M, Amato P, Sancelme M, Laj P, Leriche M, Delort AM: Contribution of microbial activity to carbon chemistry in clouds. Appl Environ Microbiol 2010, 76:23-29.

4. Hacquard S, Garrido-Oter R, Gonzalez A, Spaepen S, Ackermann G, Lebeis S, McHardy AC, Dangl JL, Knight R, Ley R, Schulze-Lefert P: Microbiota and Host Nutrition across Plant and Animal Kingdoms. Cell Host Microbe 2015, 17:603-616.

5. Zilber-Rosenberg I, Rosenberg E: Role of microorganisms in the evolution of animals and plants: the hologenome theory of evolution. FEMS Microbiol Rev 2008, 32:723-735.

6. Human Microbiome Project C: Structure, function and diversity of the healthy human microbiome. Nature 2012, 486:207-214.

7. Cho I, Blaser MJ: The human microbiome: at the interface of health and disease. Nat Rev Genet 2012, 13:260-270.

8. Epstein SS: The phenomenon of microbial uncultivability. Curr Opin Microbiol 2013, 16:636-642.

9. Group NHW, Peterson J, Garges S, Giovanni M, McInnes P, Wang L, Schloss JA, Bonazzi V, McEwen JE, Wetterstrand KA, et al: The NIH Human Microbiome Project. Genome Res 2009, 19:2317-2323.

10. Ondov BD, Treangen TJ, Melsted P, Mallonee AB, Bergman NH, Koren S, Phillippy AM: Mash: Fast genome and metagenome distance estimation using MinHash. Genome Biology 2016, 17:1-14.

11. Wainwright M, Wickramasinghe NC, Narlikar JV, Rajaratnam P: Microorganisms cultured from stratospheric air samples obtained at 41 km. FEMS Microbiol Lett 2003, 218:161165.

12. Truong DT, Franzosa EA, Tickle TL, Scholz M, Weingart G, Pasolli E, Tett A, Huttenhower C, Segata N: MetaPhlAn2 for enhanced metagenomic taxonomic profiling. Nat Methods 2015, 12:902-903.

13. Sankar A, Malone B, Bayliss SC, Pascoe B, Meric G, Hitchings MD, Sheppard SK, Feil EJ, Corander J, Honkela A: Bayesian identification of bacterial strains from sequencing data. Microb Genom 2016, 2:e000075.

14. Caporaso JG, Kuczynski J, Stombaugh J, Bittinger K, Bushman FD, Costello EK, Fierer N, Pena AG, Goodrich JK, Gordon JI, et al: QIIME allows analysis of high-throughput community sequencing data. Nat Methods 2010, 7:335-336.

15. Darling AE, Jospin G, Lowe E, Matsen FAt, Bik HM, Eisen JA: PhyloSift: phylogenetic analysis of genomes and metagenomes. PeerJ 2014, 2:e243.

16. McIntyre ABR, Ounit R, Afshinnekoo E, Prill RJ, Henaff E, Alexander N, Minot SS, Danko D, Foox J, Ahsanuddin S, et al: Comprehensive benchmarking and ensemble approaches for metagenomic classifiers. Genome Biol 2017, 18:182.

17. Breitwieser FP, Baker DN, Salzberg SL: KrakenUniq: confident and fast metagenomics classification using unique k-mer counts. Genome Biol 2018, 19:198.

18. Wood DE, Salzberg SL: Kraken: ultrafast metagenomic sequence classification using exact alignments. Genome Biol 2014, 15:R46.

19. Kim D, Song L, Breitwieser FP, Salzberg SL: Centrifuge: rapid and accurate classificaton of metagenomic sequences. Genome Research 2016, 26:1-9.

20. Nasko DJ, Koren S, Phillippy AM, Treangen TJ: RefSeq database growth influences the accuracy of k-mer-based lowest common ancestor species identification. Genome Biol 2018, 19:165.

21. Martin TC, Visconti A, Spector TD, Falchi M: Conducting metagenomic studies in microbiology and clinical research. Appl Microbiol Biotechnol 2018, 102:8629-8646.

22. Tatusova T, Ciufo S, Federhen S, Fedorov B, McVeigh R, O'Neill K, Tolstoy I, Zaslavsky L: Update on RefSeq microbial genomes resources. Nucleic Acids Res 2015, 43:D599-605. 
702 23. Balvociute M, Huson DH: SILVA, RDP, Greengenes, NCBI and OTT - how do these 
Table 1. Description of 7 classification indices used in this study

\begin{tabular}{|c|c|c|c|c|}
\hline Index name & $\begin{array}{l}\text { Reference database and } \\
\text { taxonomic definitions used }\end{array}$ & Description & $\begin{array}{l}\text { Total number of } \\
\text { reference genomes } \\
\text { included }\end{array}$ & $\begin{array}{c}\text { Strictly } \\
\text { monophyletic } \\
\text { species } \\
\text { definitions }\end{array}$ \\
\hline NCBI_r86 & NCBI RefSeq release 86 & Complete microbial genomes (r86) & 8674 & $\mathrm{~N}$ \\
\hline GTDB_r86_8.6k & GTDB release 86 & $\begin{array}{l}\text { Same genomes as NCBI_8.6k but with GTDB } \\
\text { taxonomic definitions, to compare effect of strict } \\
\text { monophyletic definitions }\end{array}$ & 8674 & $\mathrm{Y}$ \\
\hline NCBI_r88 & NCBI RefSeq release 88 & Complete microbial genomes (r88) & 10089 & $\mathrm{~N}$ \\
\hline NCBI_r88_Human17k & NCBI RefSeq release 88 & $\begin{array}{l}\text { Same as NCBI_r88 with the addition of all draft } \\
\text { genomes from } 70 \text { genera of interest, strictly corrected } \\
\text { for monophyly }\end{array}$ & 16908 & $\begin{array}{l}\text { Y (for } 70 \text { genera } \\
\text { only) }\end{array}$ \\
\hline GTDB_r86_noMAGs & GTDB release 86 & $\begin{array}{l}\text { GTDB r86 without metagenome-assembled genomes } \\
\text { (MAGs) }\end{array}$ & 25660 & Y \\
\hline GTDB_r86 & GTDB release 86 & $\begin{array}{l}\text { All dereplicated* bacterial and archaeal genomes used } \\
\text { to curate the GTDB taxonomy in the GTDB study }\end{array}$ & 28560 & $\mathrm{Y}$ \\
\hline GTDB_r86_46k & GTDB release 86 & $\begin{array}{l}\text { Manual dereplication* of GTDB release } 86 \text { to get more } \\
\text { bacterial and archaeal reference genomes with GTDB } \\
\text { taxonomic definitions }\end{array}$ & 46006 & $\mathrm{Y}$ \\
\hline
\end{tabular}

* Dereplication is defined as the threshold-based selection of representative reference genomes for phylogenetically-similar clusters. 


\section{Legend:}

$\begin{array}{llcllc}\text { Index name } & \text { Taxonomic definitions } & \text { \# genomes } & \text { Index name } & \text { Taxonomic definitions } & \text { \# genomes } \\ \text { NCBI_r86 } & \text { Default NCBI RefSeq r86 } & 8,674 & \text { GTDB_r86_noMAGs } & \text { GTDB_r86 without MAGs } & 25,660 \\ \text { GTDB_r86_8.6k } & \text { NCBI_r86 with GTDB definitions } & 8,674 & \text { GTDB_r86 } & \text { Representative genomes from GTDB } & 28,560 \\ \text { NCBI_r88 } & \text { Default NCBI RefSeq r88 } & 10,089 & \text { 由 GTDB_r86_46k } & \text { GTDB_r86 + more genomes / taxon } & 46,006 \\ \text { NCBI_r88_Human17k } & \begin{array}{l}\text { NCBI_r88 + 70 corrected human- } \\ \text { associated genera }\end{array} & 16,908 & & \end{array}$
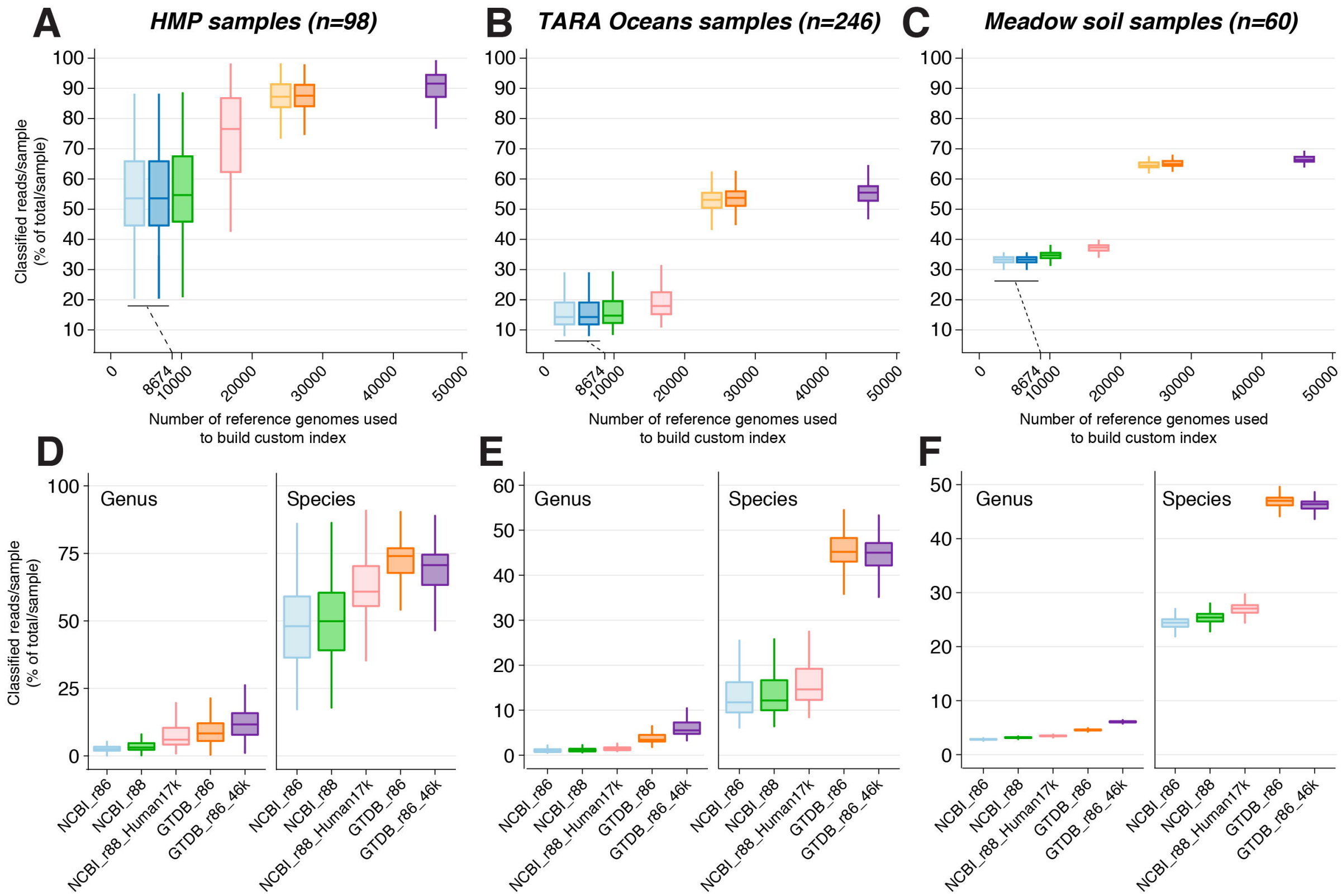


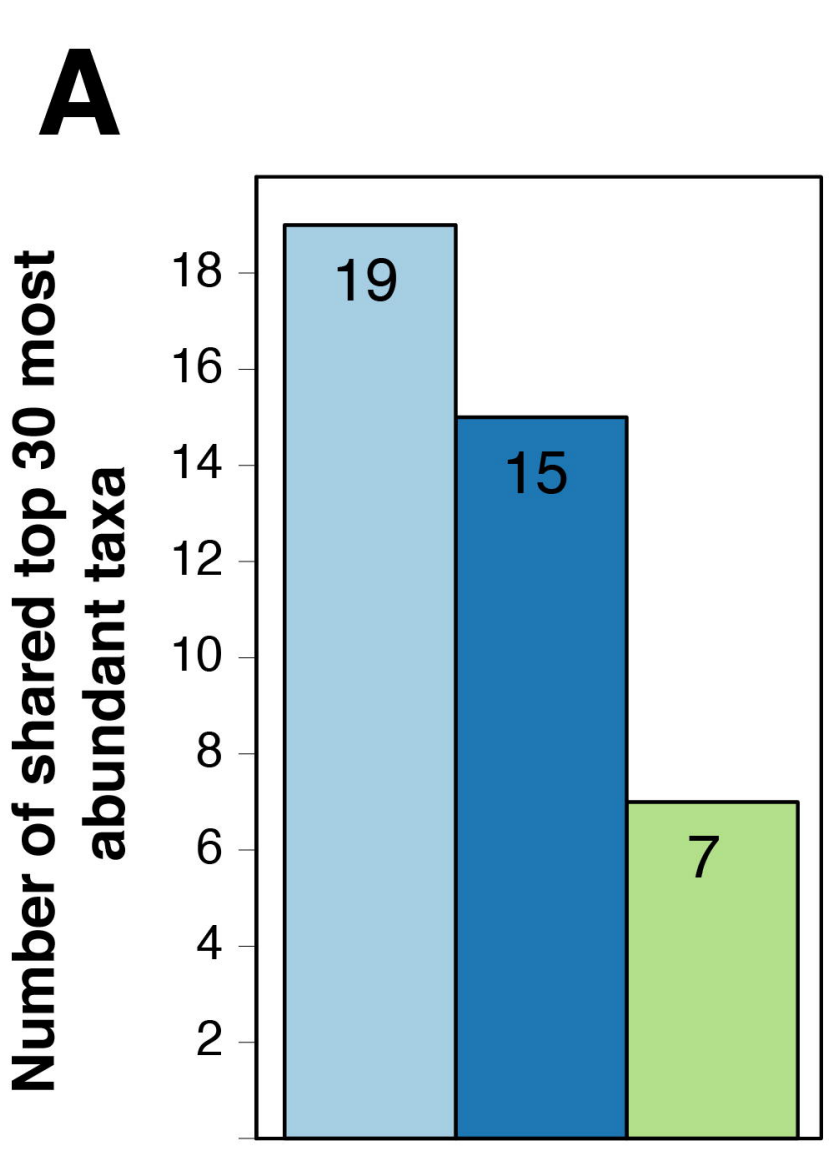

B

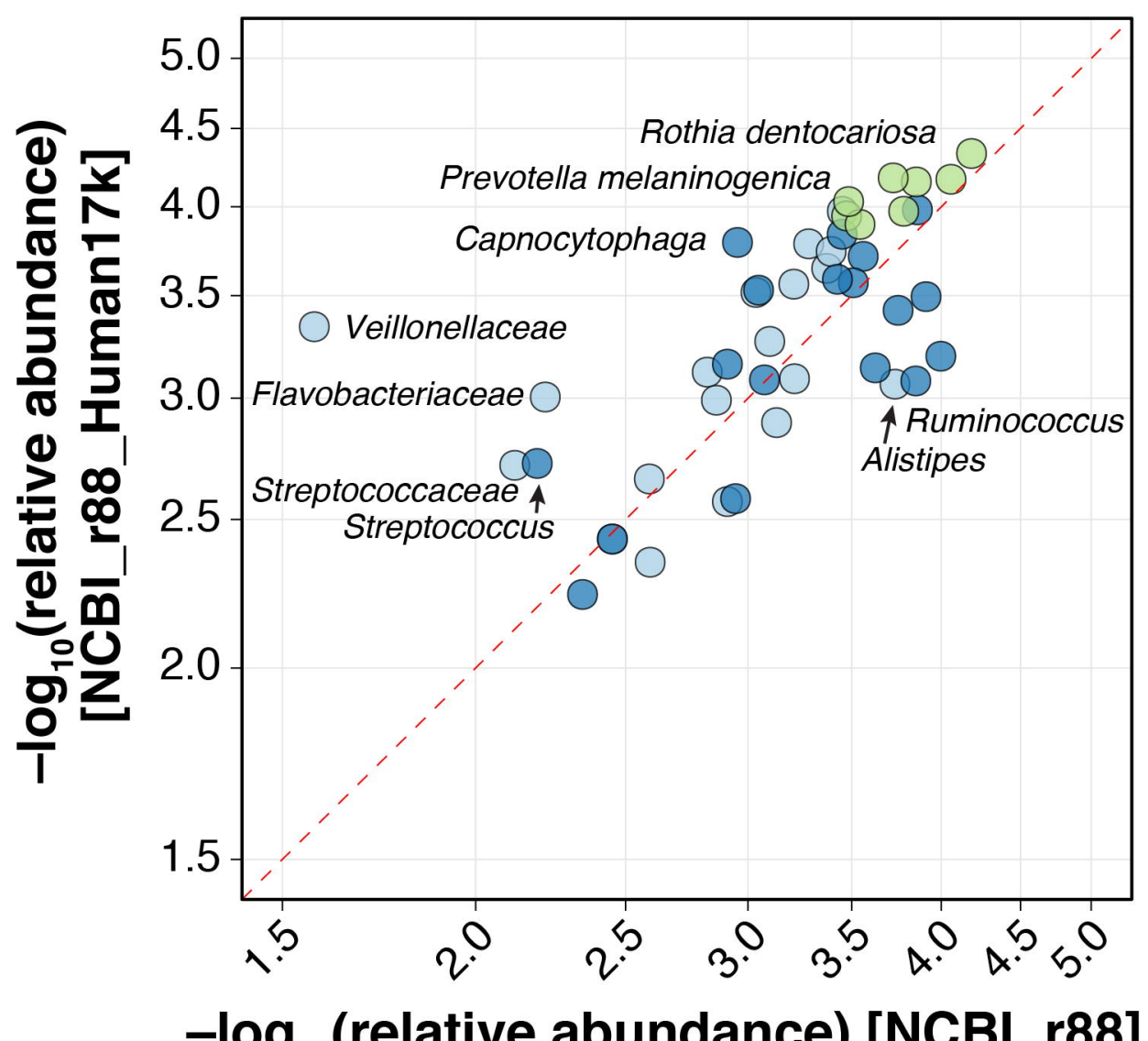

- $\log _{10}$ (relative abundance) [NCBI_r88]

Spearman $_{\text {ALL }} \rho=0.691\left(p=1.46 \times 10^{-7}\right)$

Spearman $_{F} \rho=0.610\left(p=6.52 \times 10^{-3}\right)$

Spearman $_{\mathrm{G}} \rho=0.463(p=0.054)$

Spearman $_{s} \rho=0.714(p=0.057)$
Taxonomic rank of $\bigcirc$ Family $(n=19)$

shared taxon: Genus $(n=15)$

Species $(n=7)$

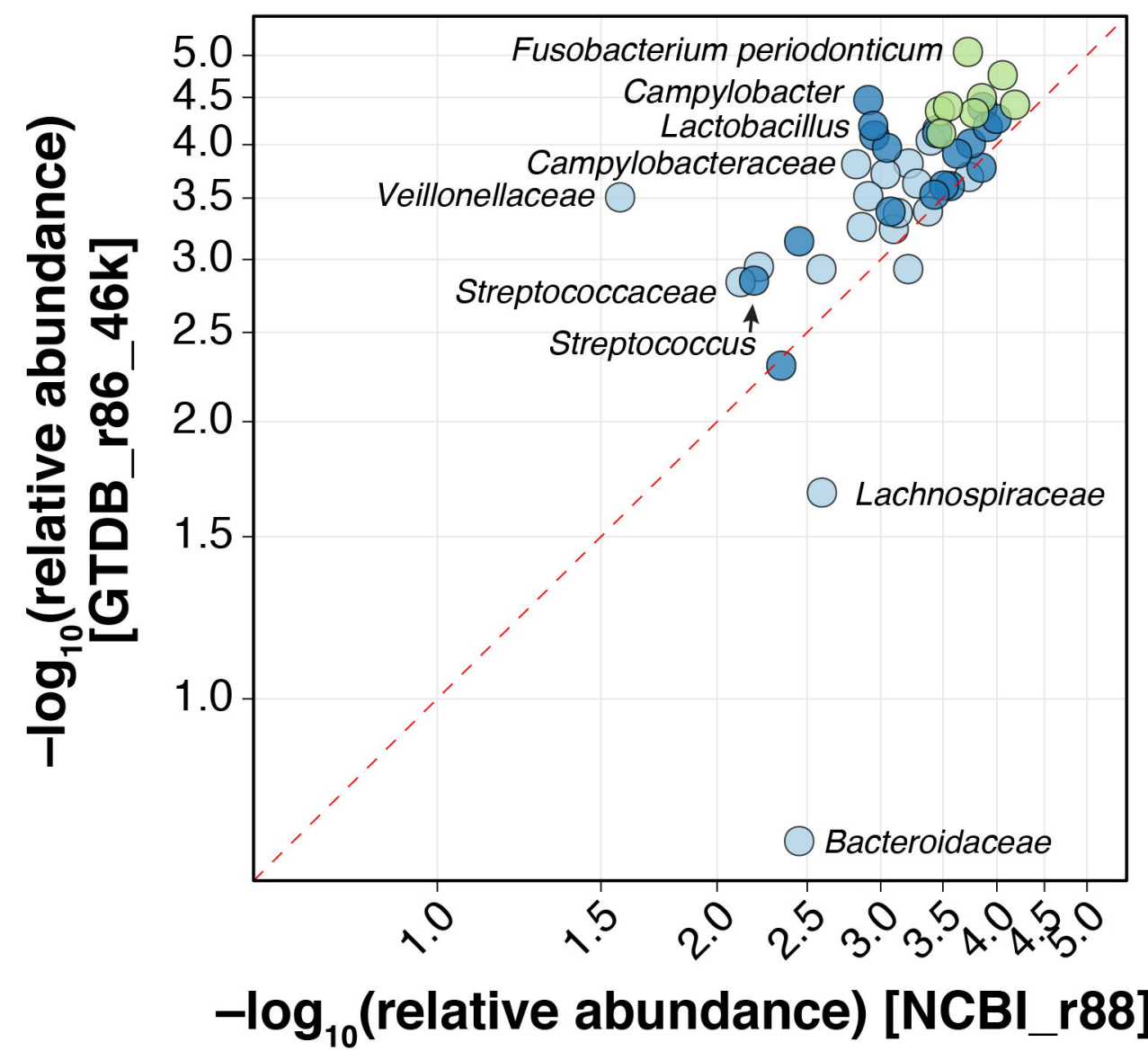

Spearman $_{\text {ALL }} \rho=0.724\left(p=1.85 \times 10^{-8}\right)$

Spearman $_{F} \rho=0.691\left(p=4.29 \times 10^{-3}\right)$

Spearman $_{G} \rho=0.459(p=0.056)$ Spearman $_{s} p=0.524(p=0.196)$ 
Figure 3.
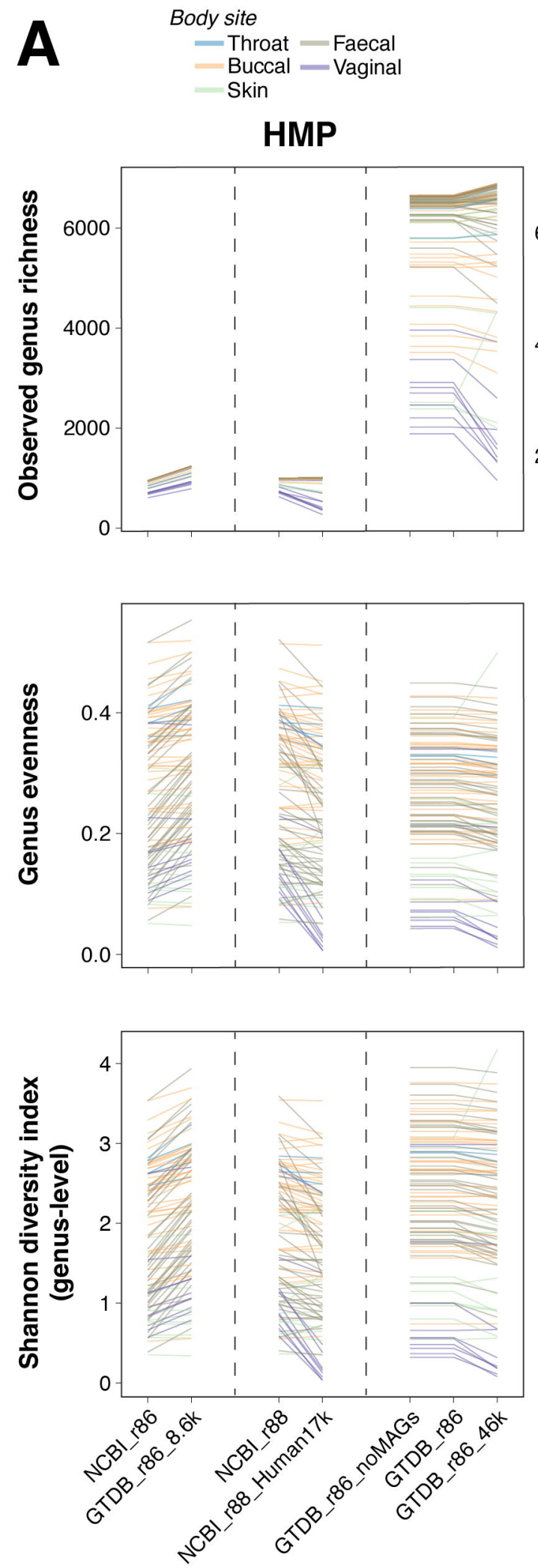

Sampling depth Deep chlorophyll max (DCM) - Mesopelagic (MESO)

TARA Oceans
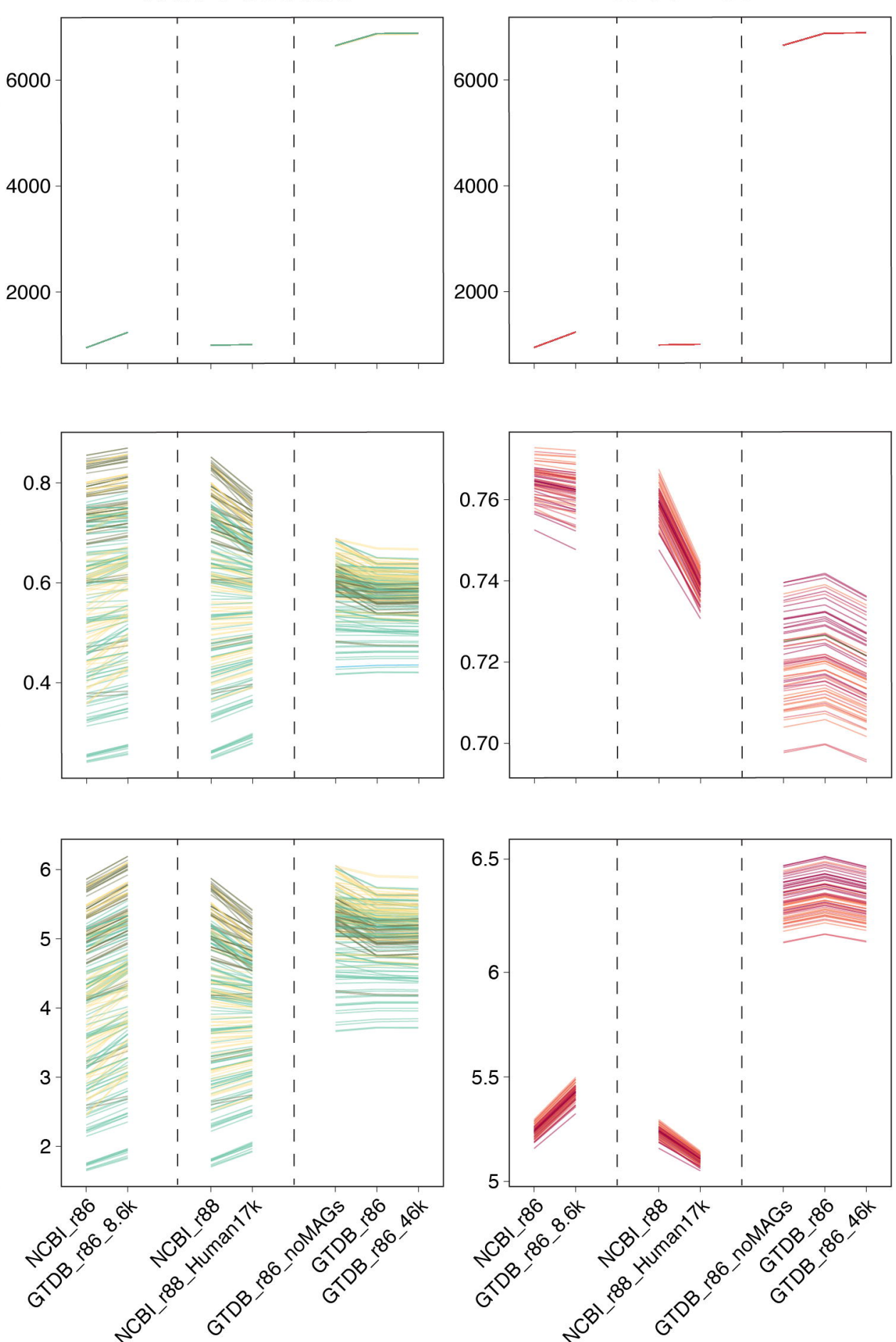

Sampling depth

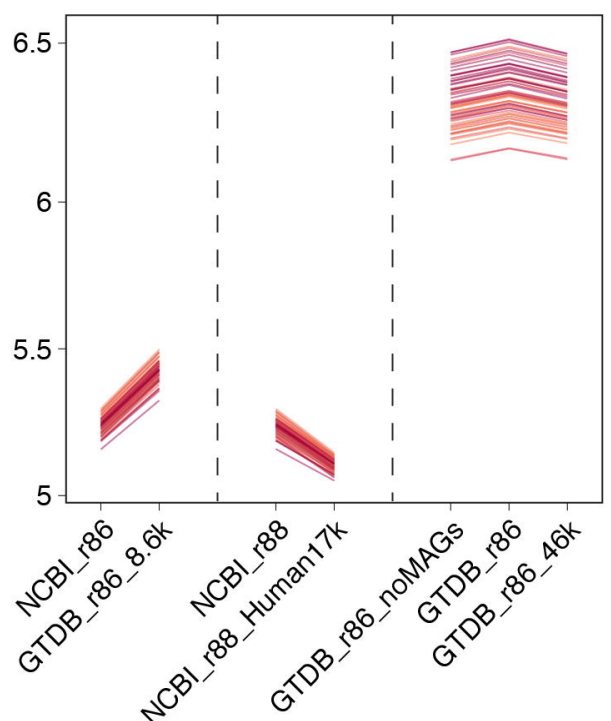

B

Metagenomic dataset HMP

TARA Ocean

Meadow soil
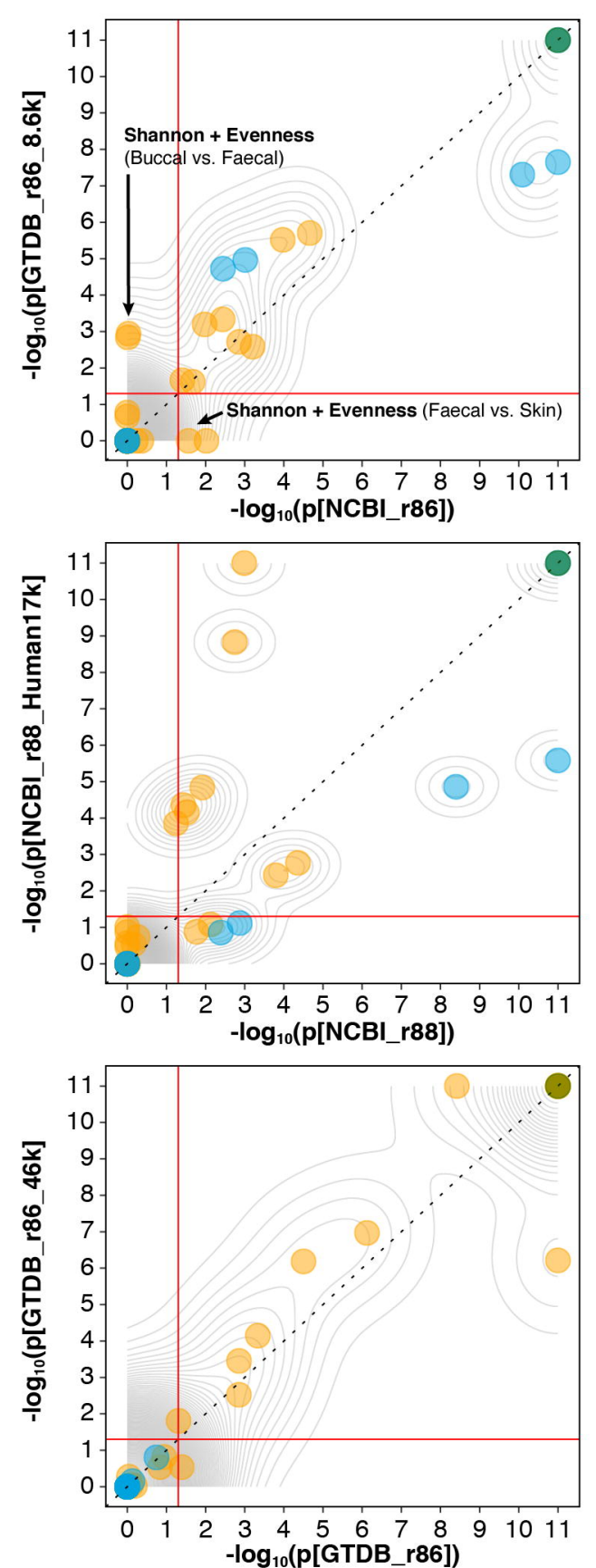
Figure 4.

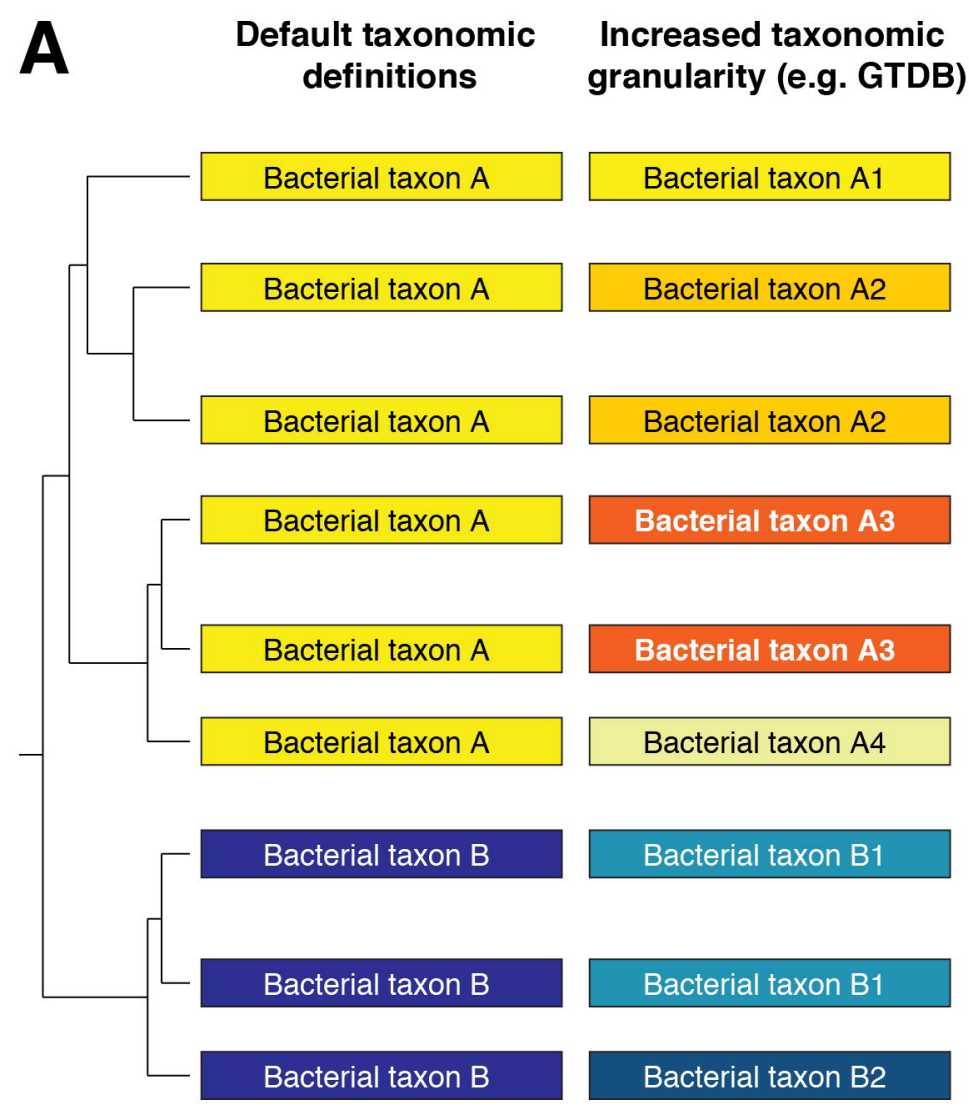

\section{B Metagenomic classification with index database built using:} $\begin{array}{cc}\text { Default reference genomes } & \text { More reference genomes } \\ \text { and default taxonomic } & + \text { increased taxonomic }\end{array}$ + increased taxonomic
granularity (e.g. GTDB)
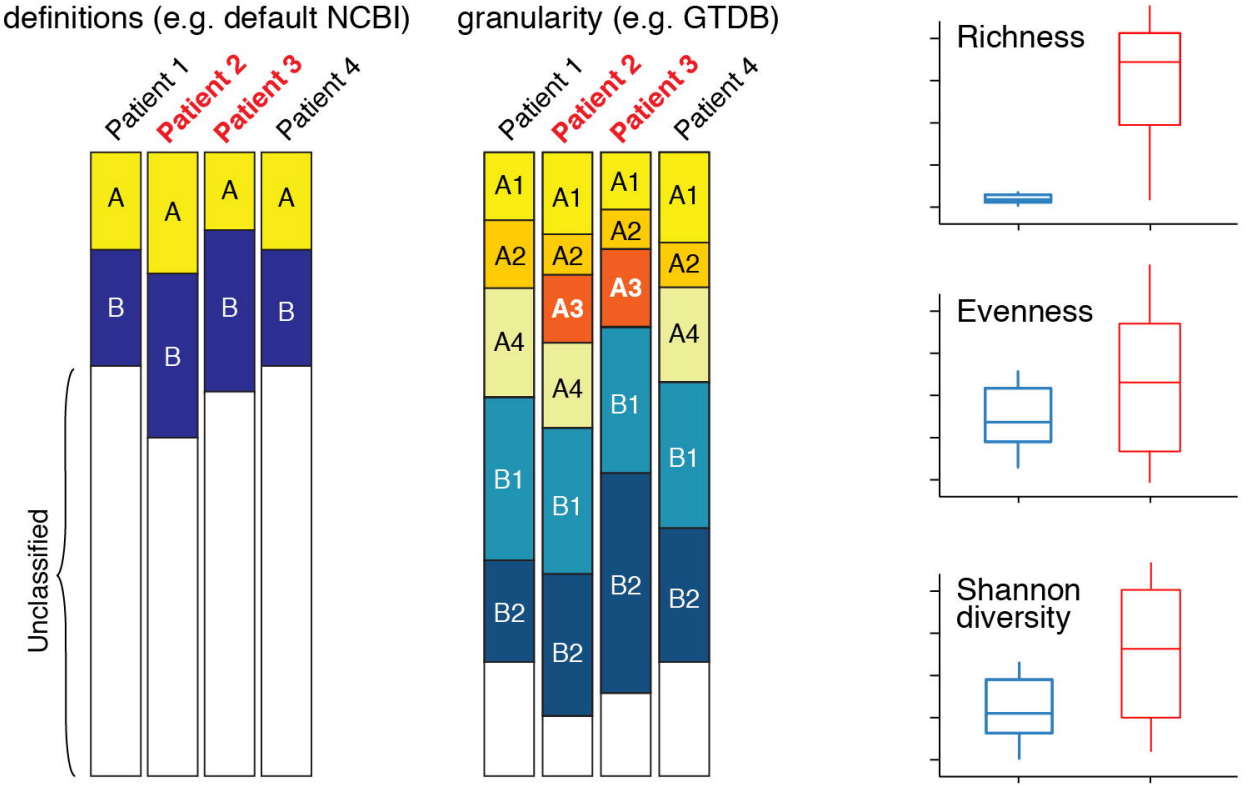
$\longrightarrow$ Impact on analyses based on: - composition data (relative abundance) - diversity metrics (alpha, beta)

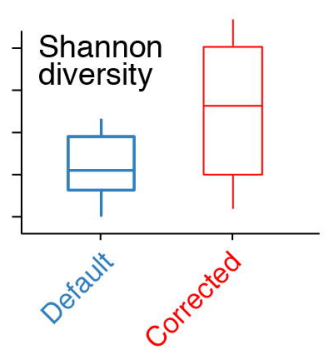

C Impact of index database correction on downstream analyses: Diversity analyses:

Microbiome-wide association studies (MWAS) Q-Q plot:

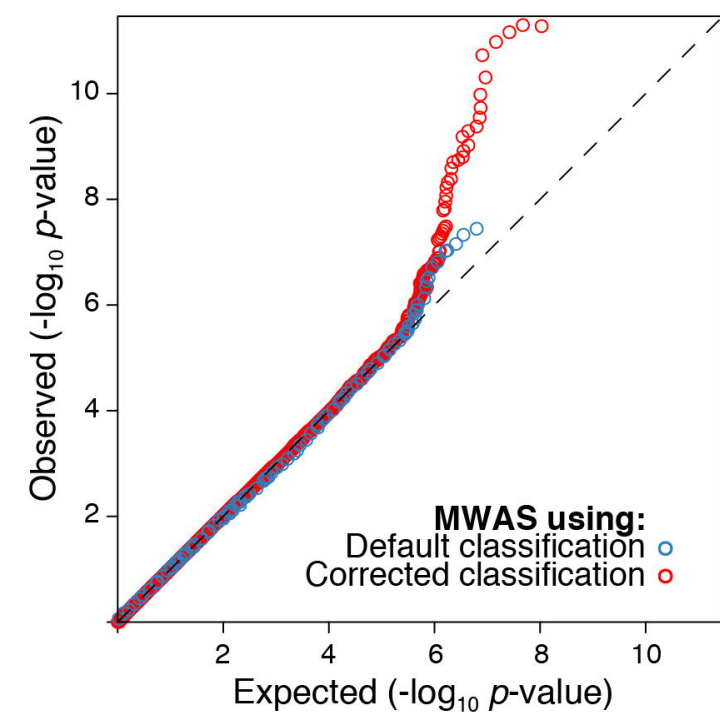

Expected $\left(-\log _{10} p\right.$-value) 


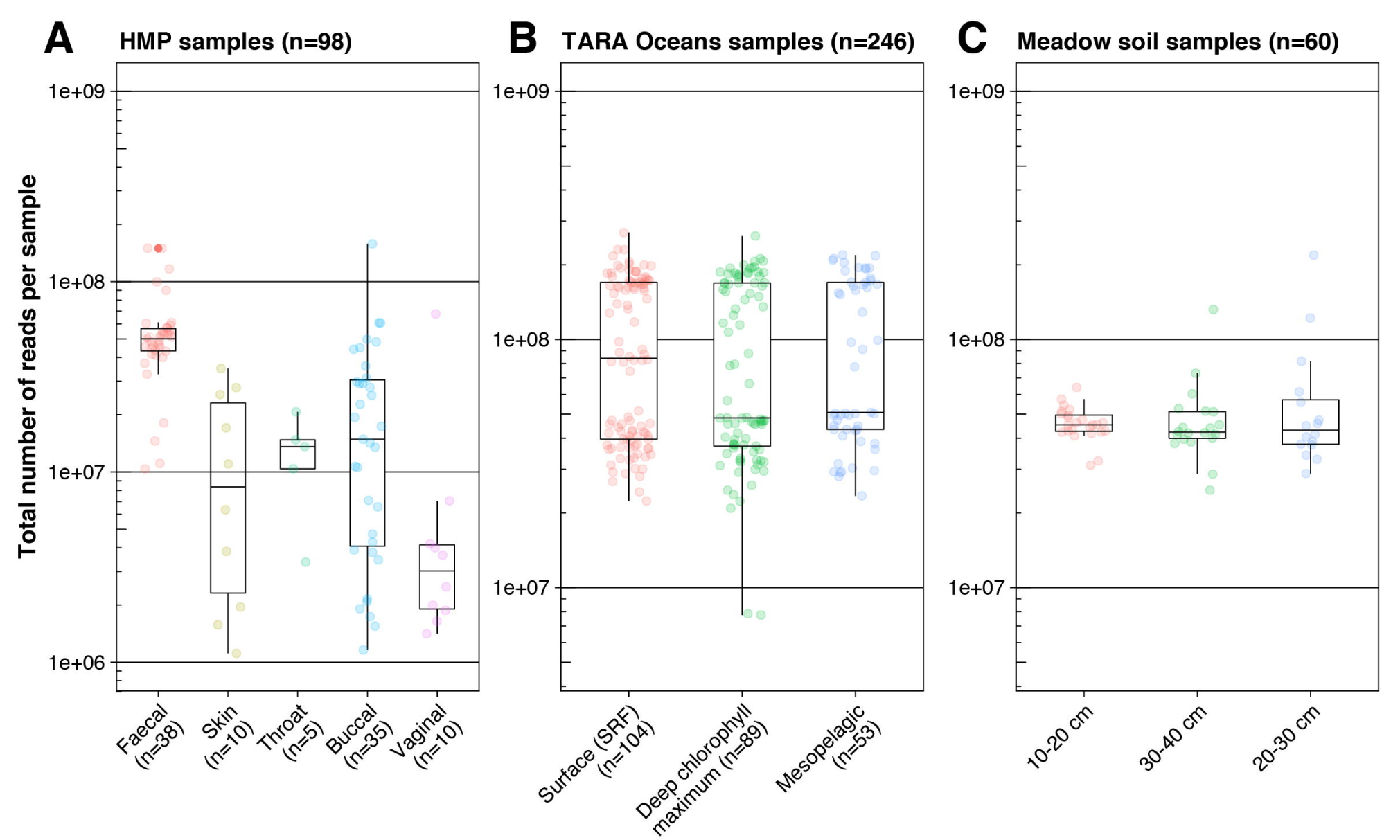




\section{Figure S2.}

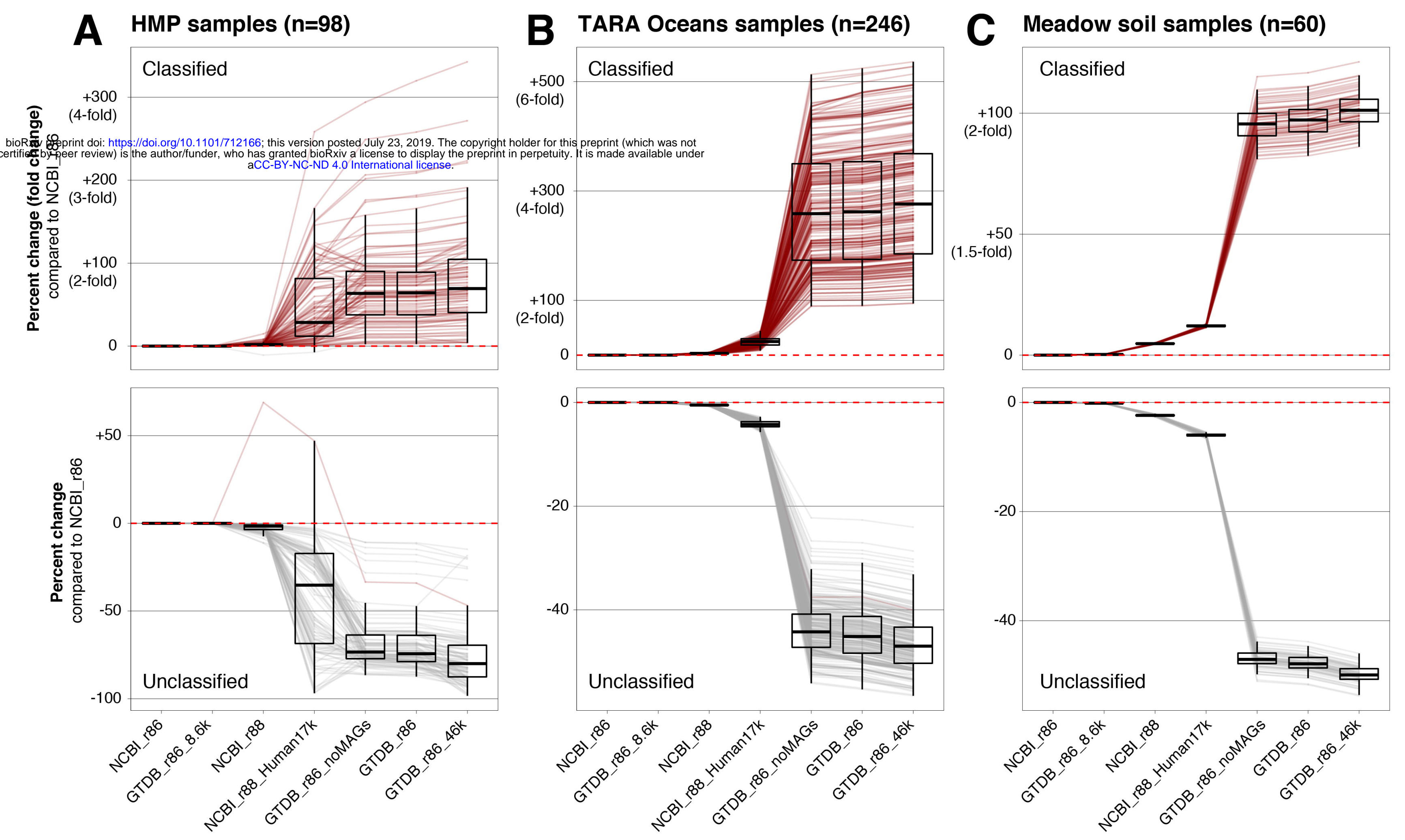




\section{Figure S3.}

A. HMP samples $(n=98)$

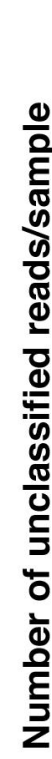

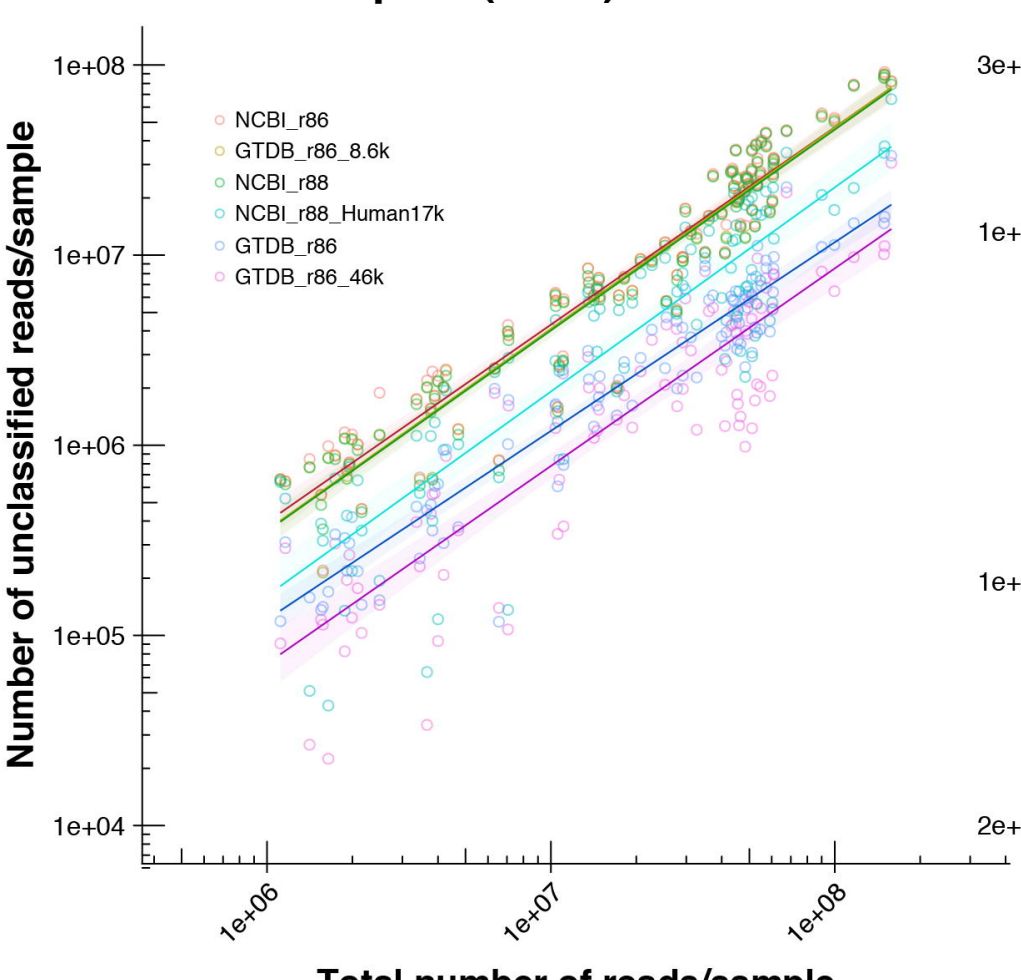

Total number of reads/sample
B. TARA Oceans samples $(n=246)$

$2 \mathrm{e}+06$

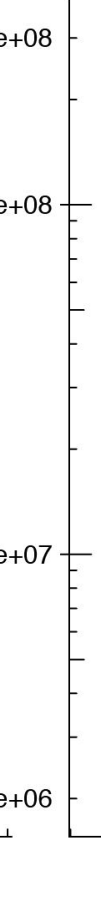

NCBI_r86

GTDB_r86_8.

NCBI_r88

GTDB_86

GTDB_r86_46k

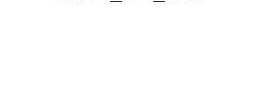

,

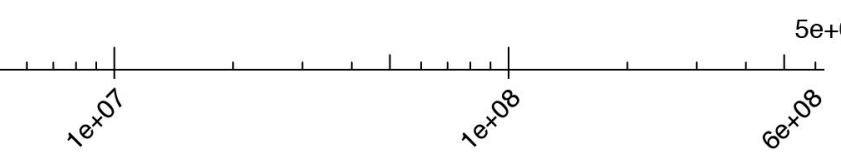

Total number of reads/sample
C. Meadow soil samples $(n=60)$

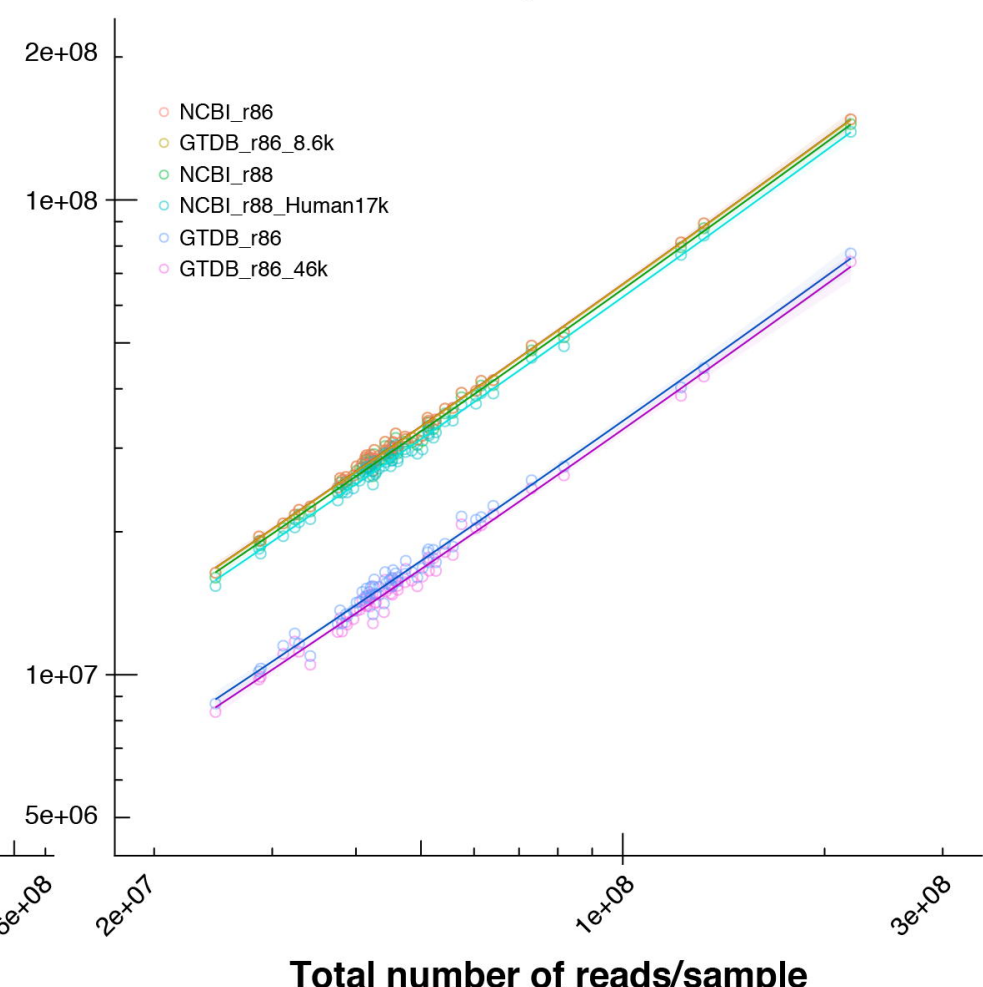


doi: https://doi.org/10.1101/712166; this version posted July 23, 2019. The copyright holder for this preprint (which was not

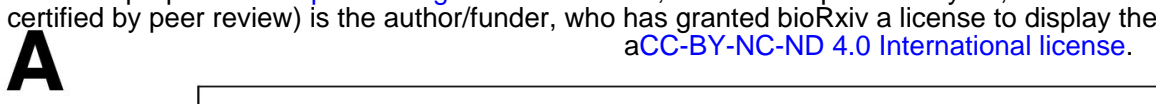
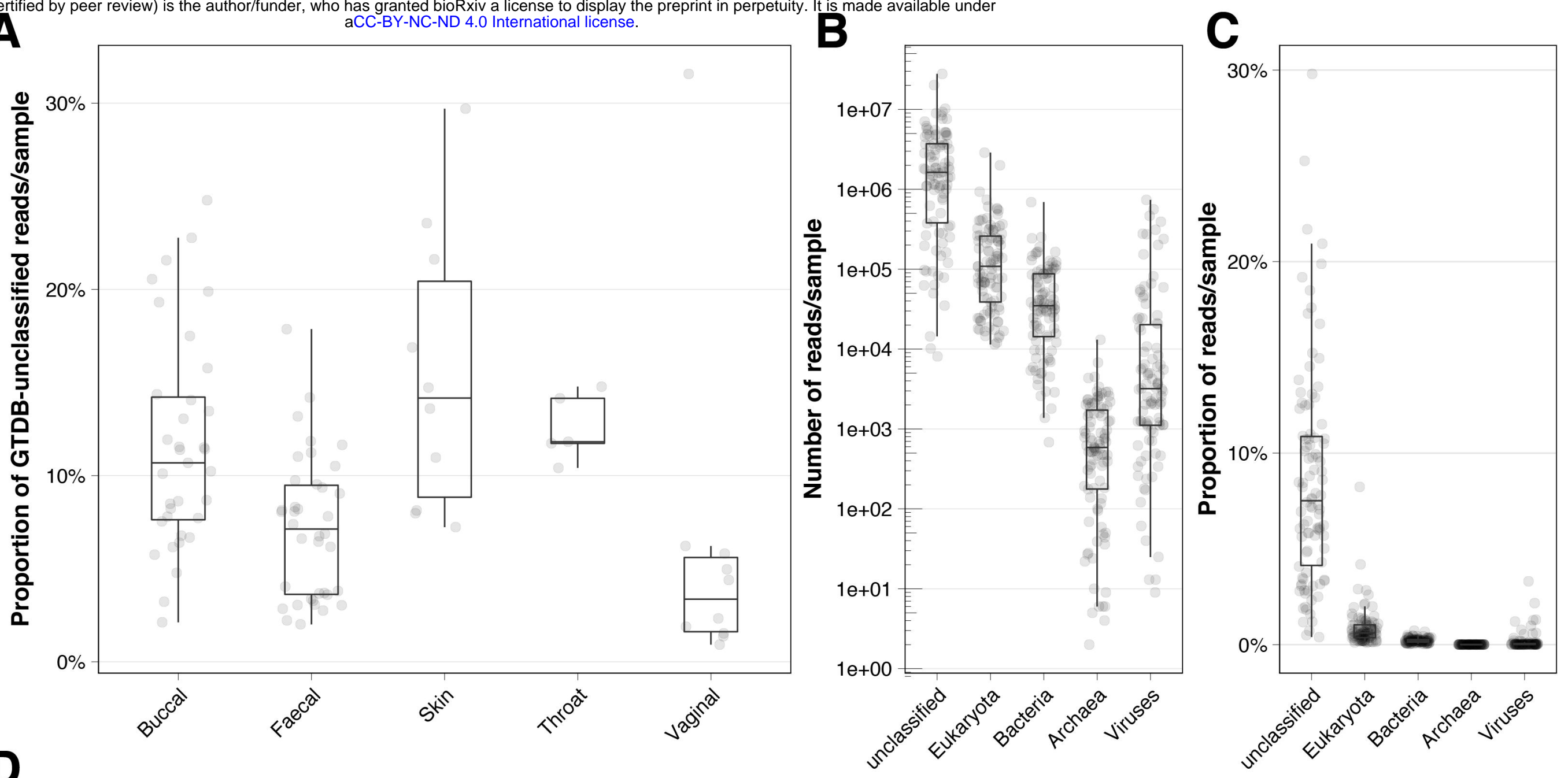

D
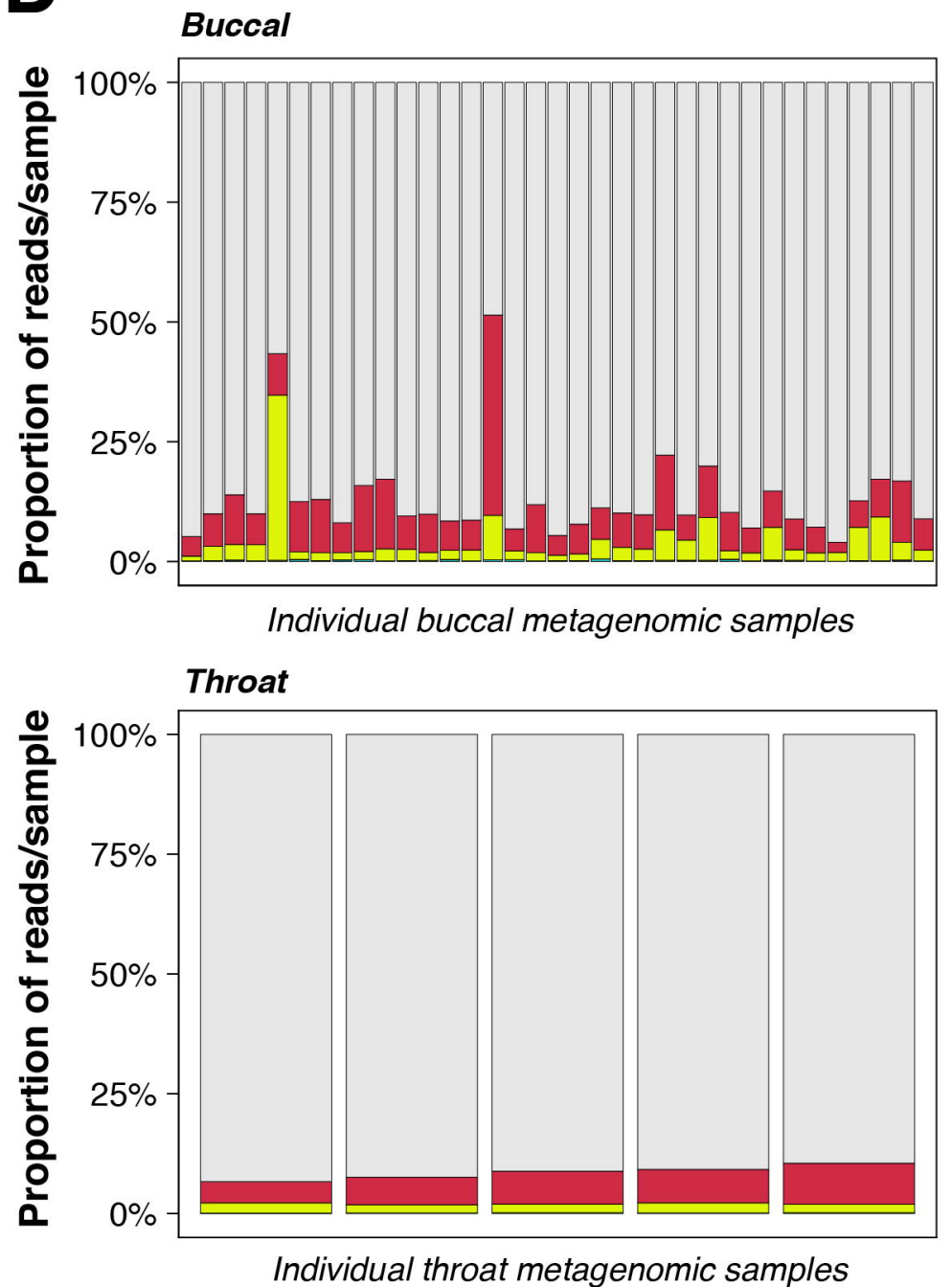

Faecal

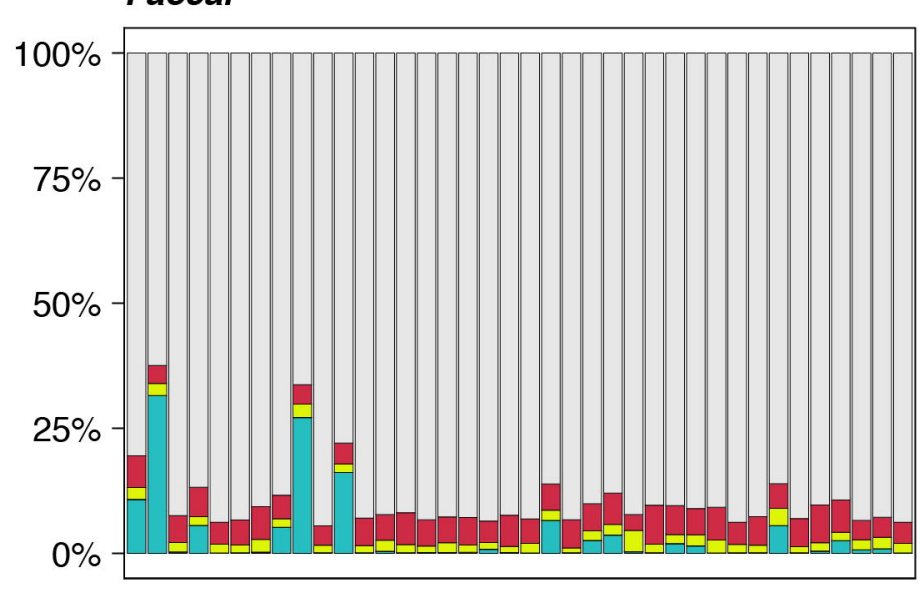

Individual faecal metagenomic samples

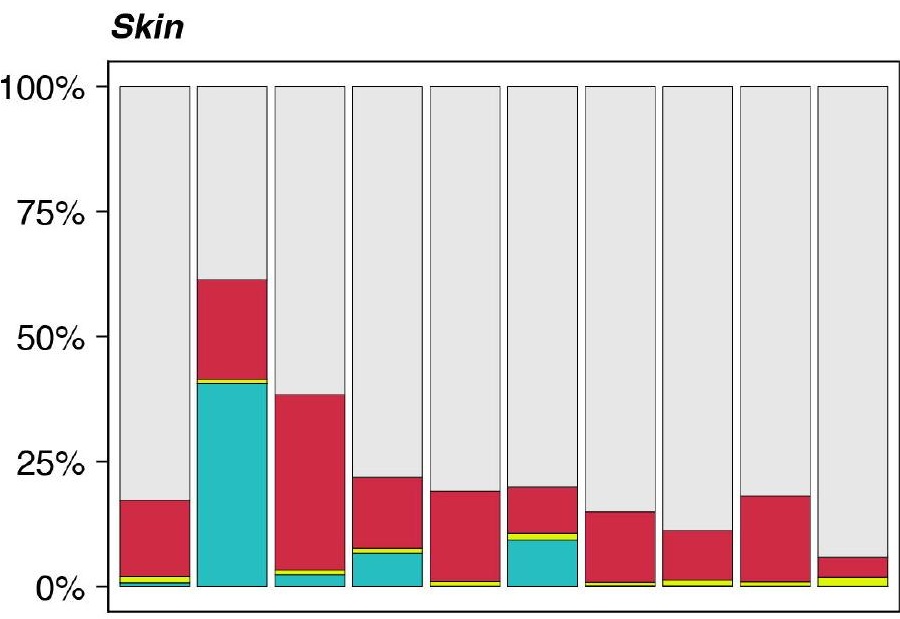

Individual skin metagenomic samples
Unclassified

Eukaryota

Bacteria

Archaea

Viruses 
A

Genus corrected in NCBI_r88_Human17k

Becterides

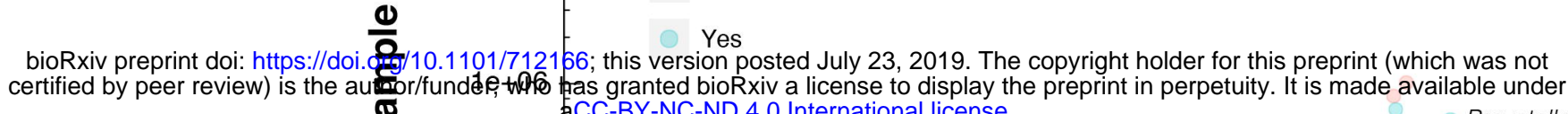

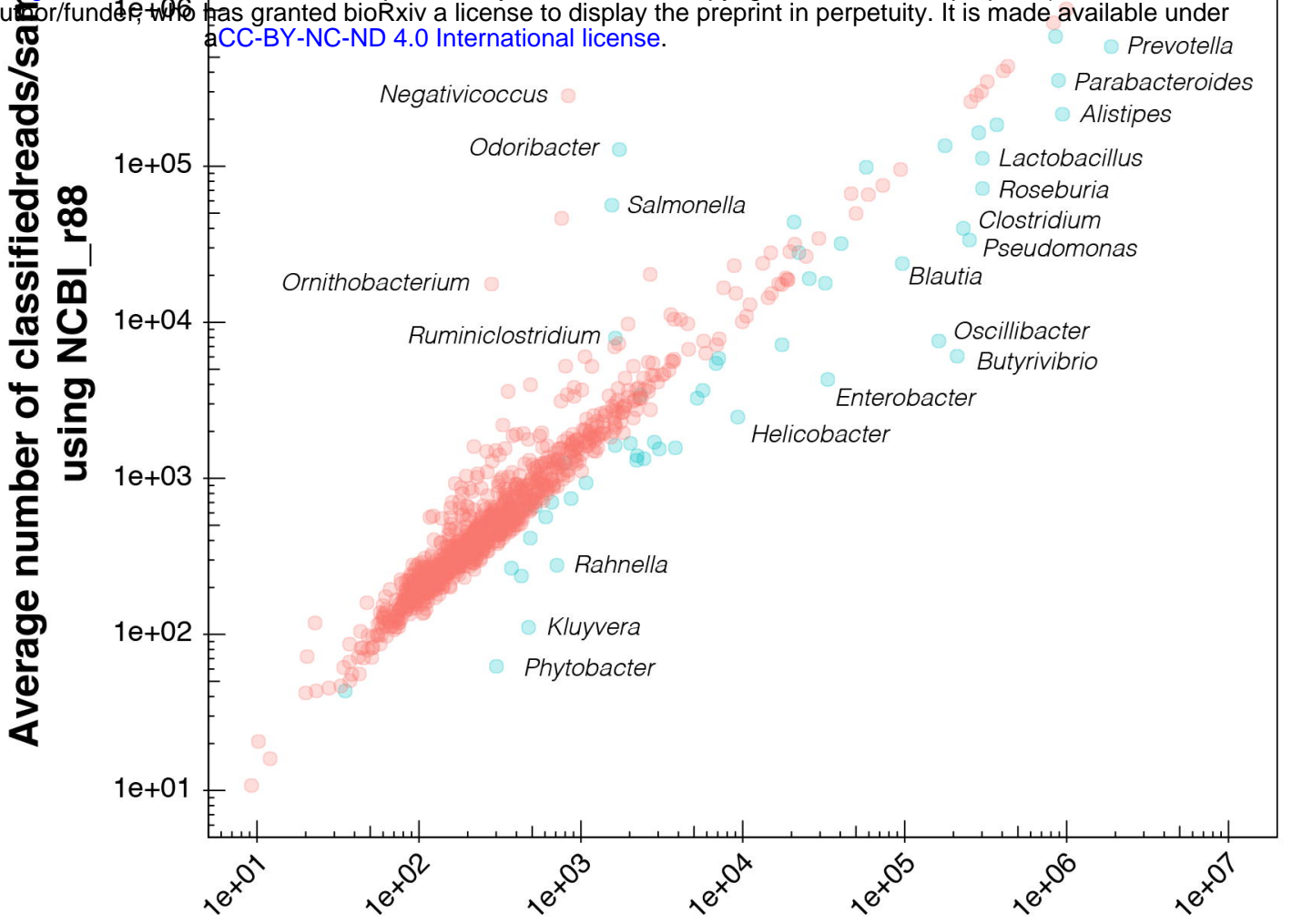

Average number of classified reads/sample using NCBI_r88_Human17k

C

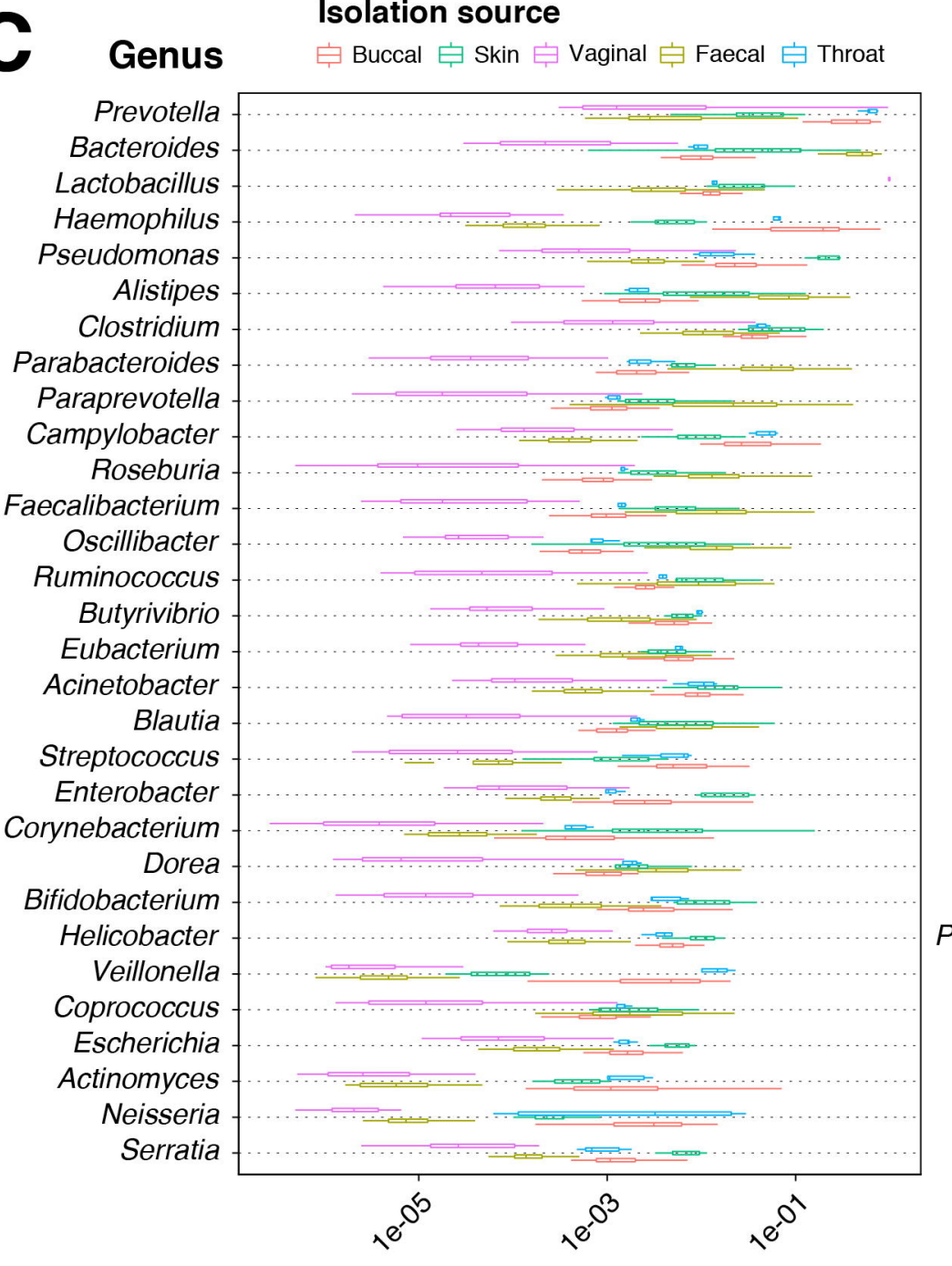

Proportion of classified reads using NCBI r88 Human17k from unclassified reads using NCBI_r88 using NCBI_r88

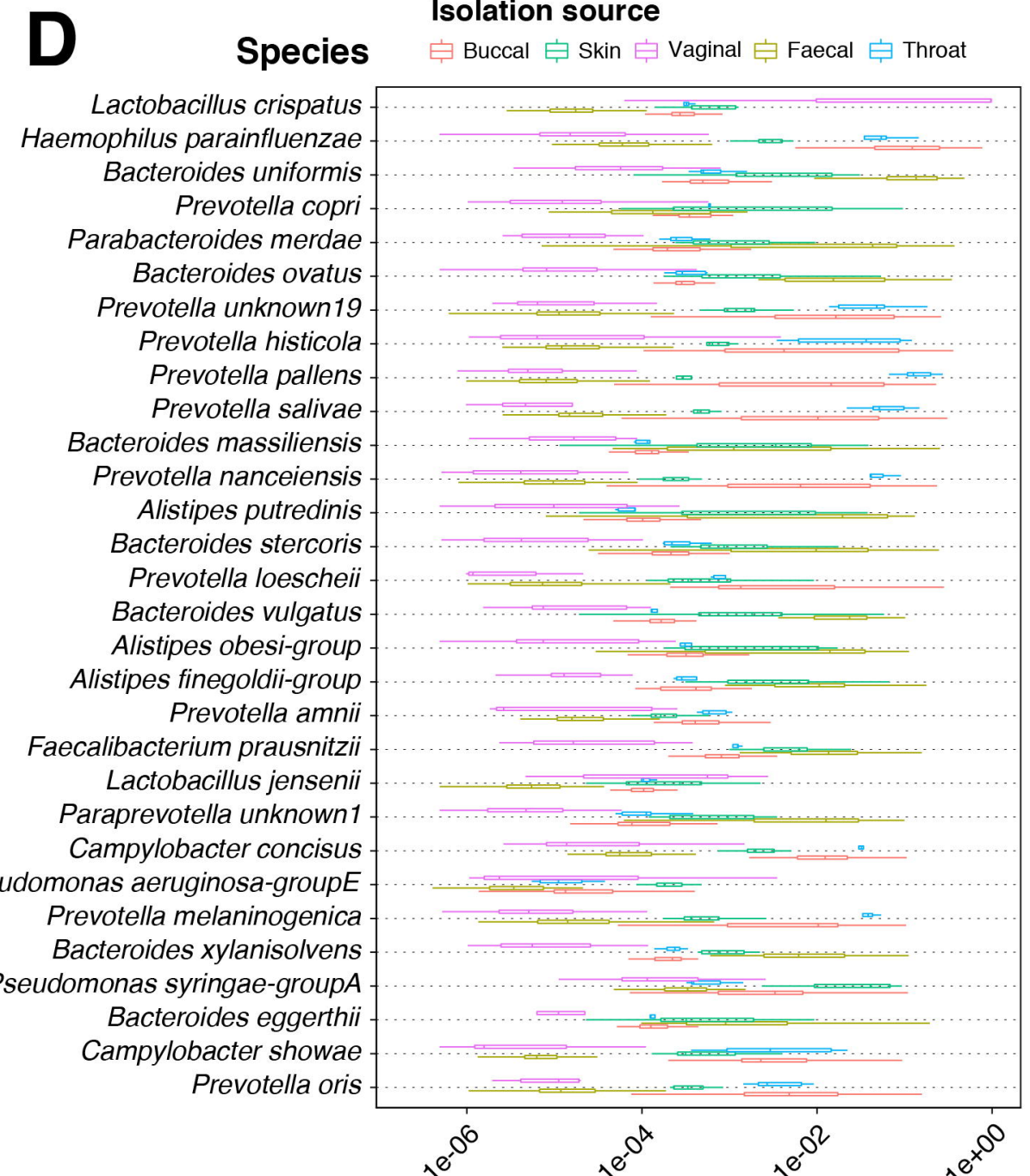

Proportion of classified reads using NCBI_r88_Human17k from unclassified reads
B HNCBI_r88 NCBI_r88_Human17k

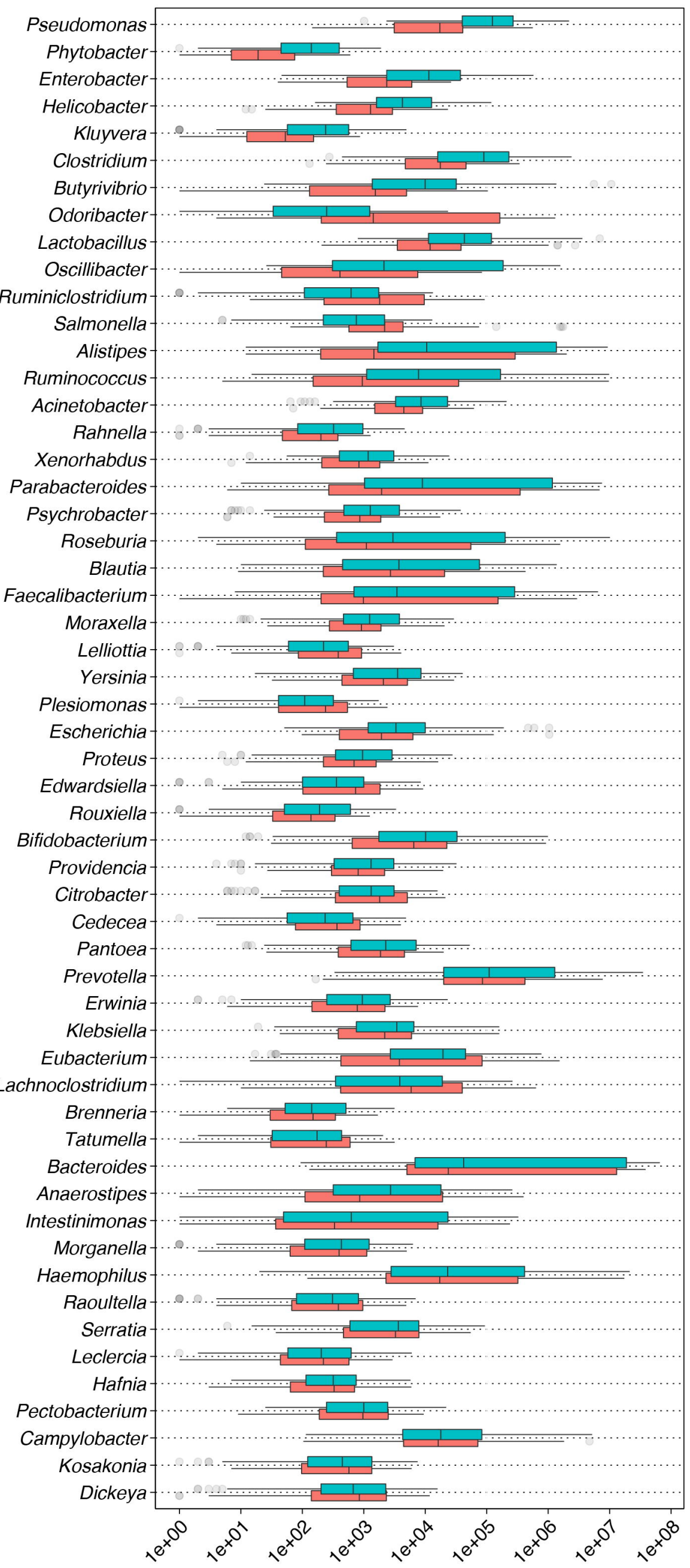

Number of classified HMP reads 


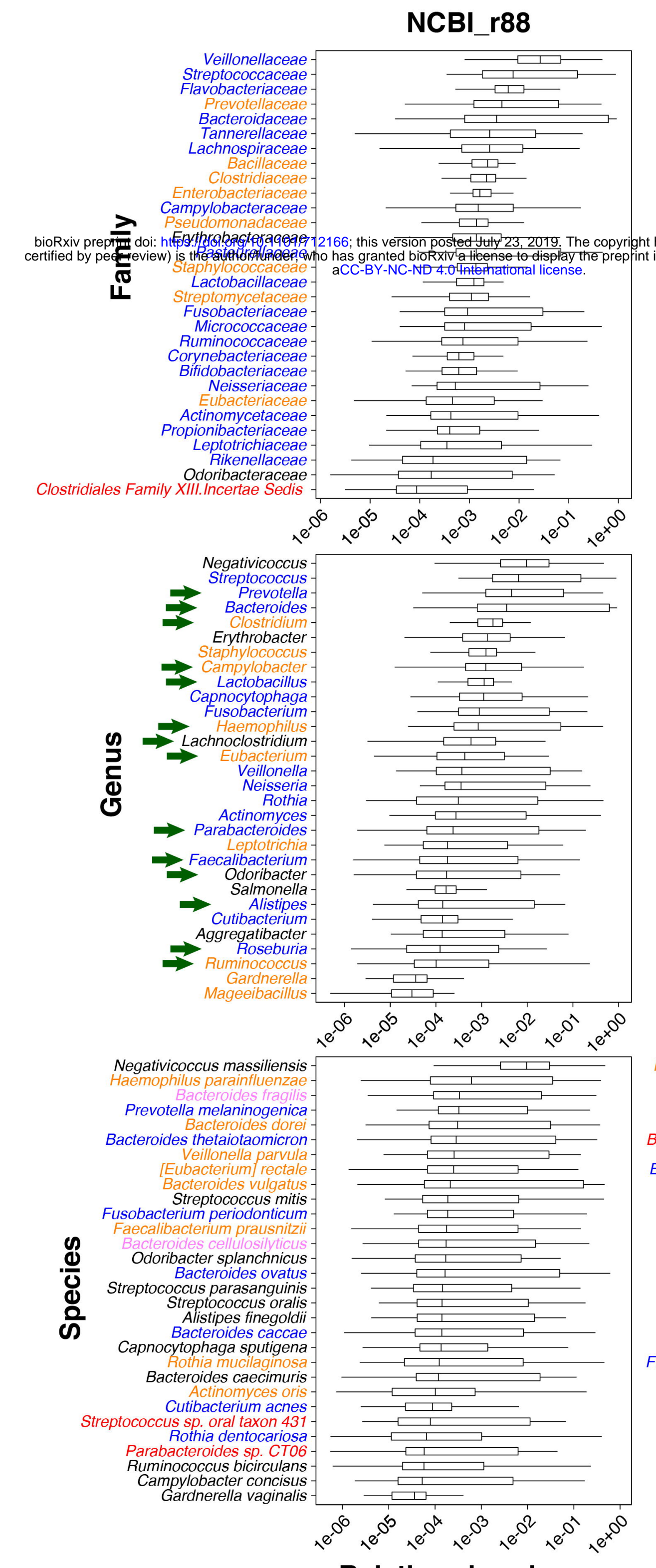

Relative abundance
NCBI_r88_Human17k
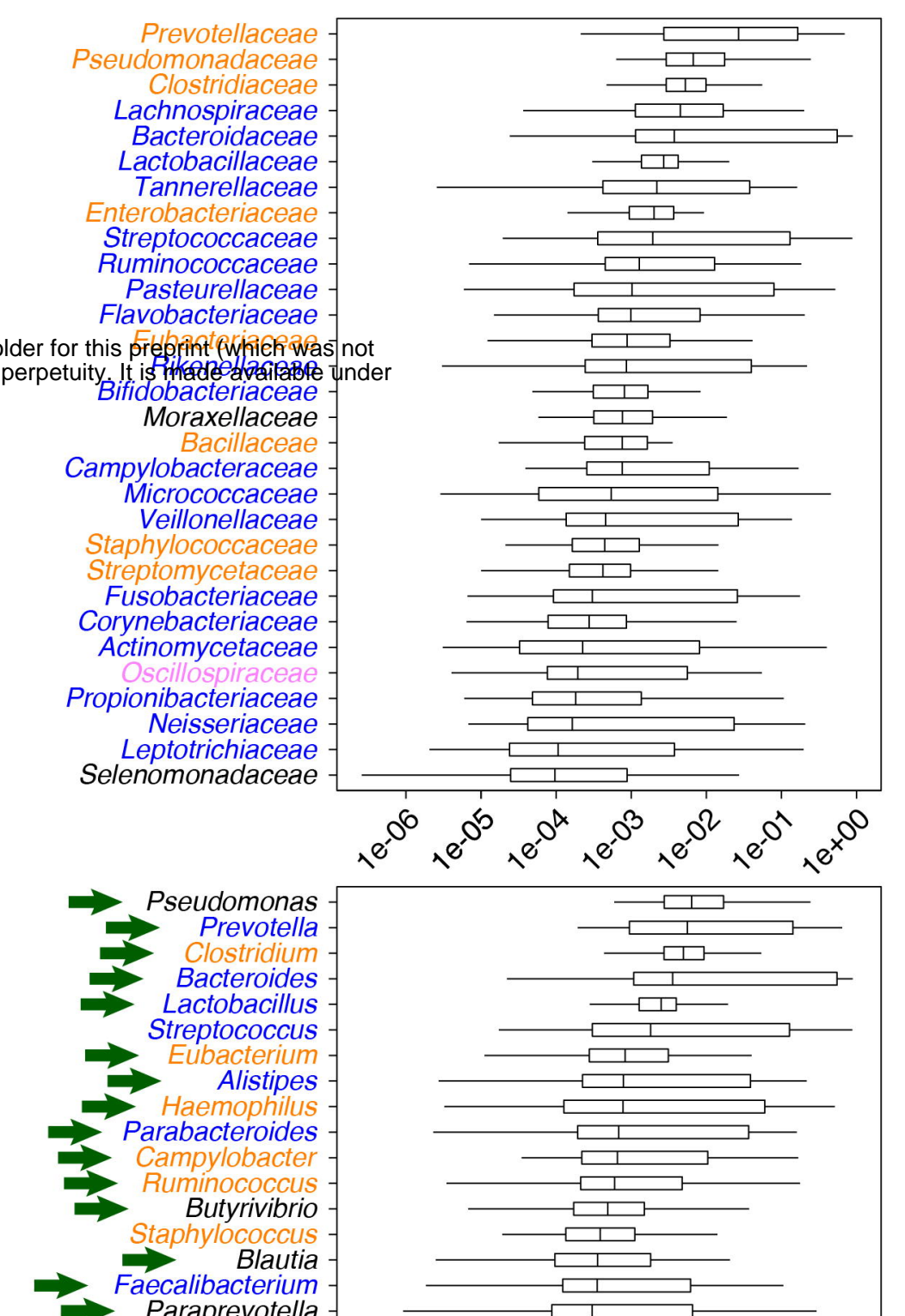

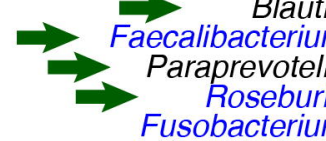

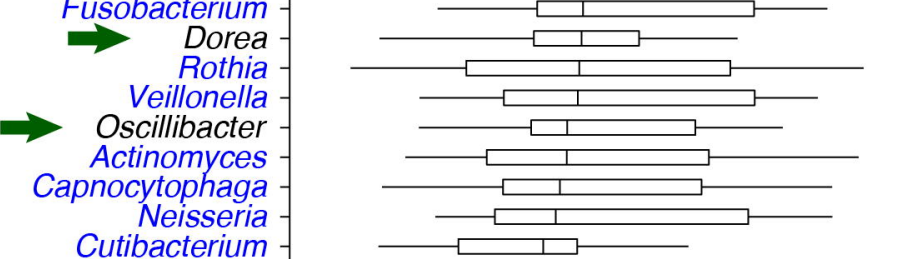

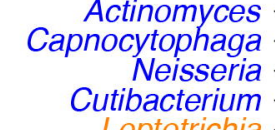

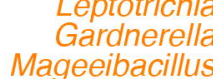
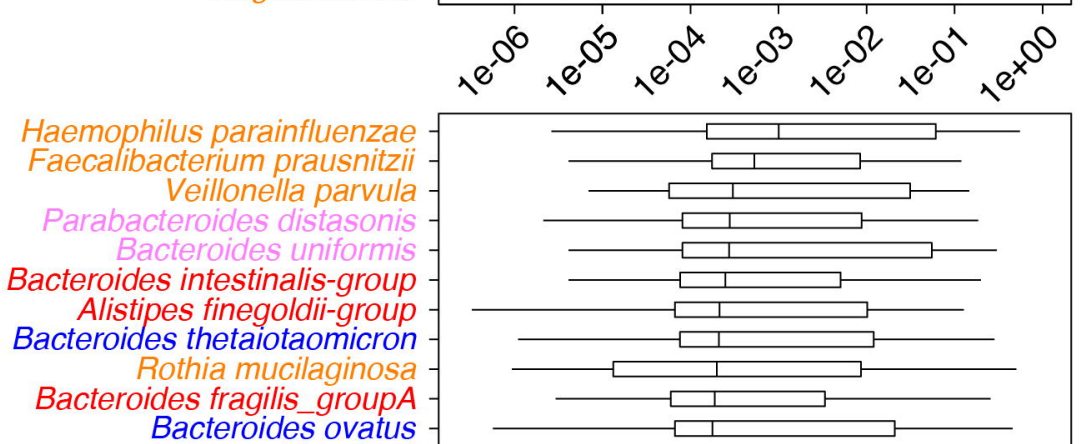

Prevotetlla melanininoegonicica

Bactereides stercoris
Bacteroides caccae

Fusobacteriumacteritiod acticum

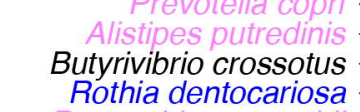

Bacteroidies massiliensis
Paraarevotella unknownt

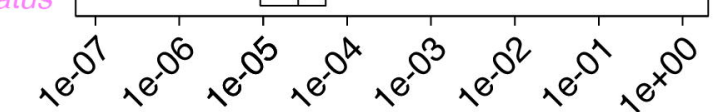

Relative abundance
GTDB_r86_46k

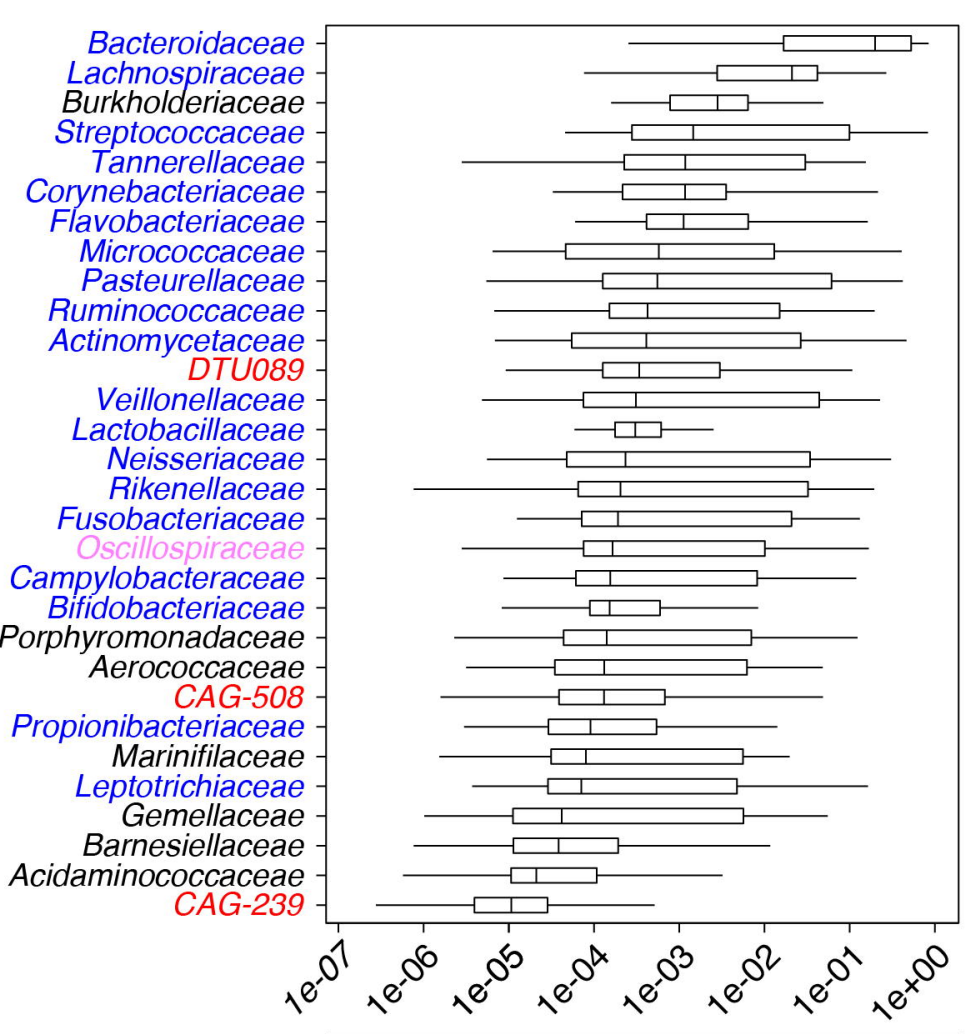

Family amongst top 30 most abundant families in:

One index only

NCBI_r88 and NCBL r88_17k only $(n=8)$

All 3 indices $(n=19)$

Family definition unique to index

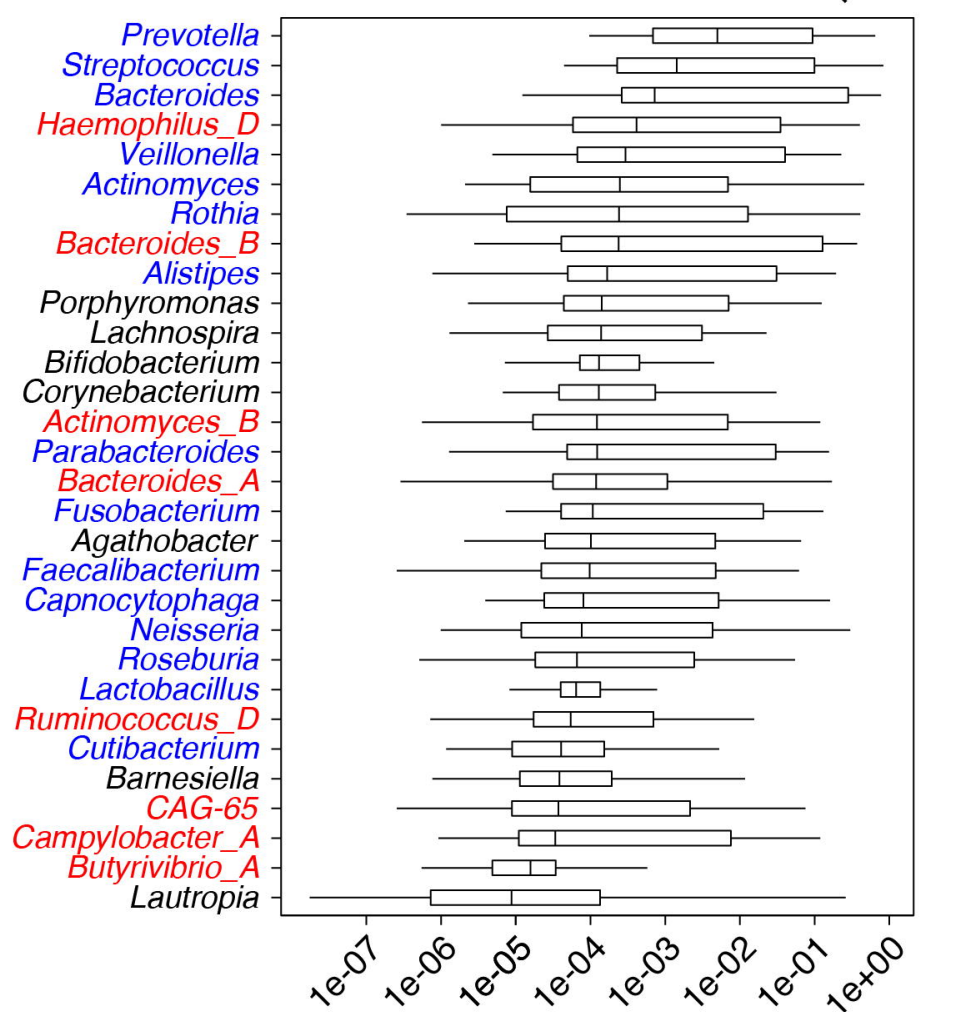

Genus amongst top $\mathbf{3 0}$ most abundant genera in:

One index only

NCBI r88 and NCBI_r88_17k only (n=9)

GTDB_r86_46K all 3 indices $(n=15)$

Genus definition unique to index

- Genus corrected in NCBI_r88_17k customization

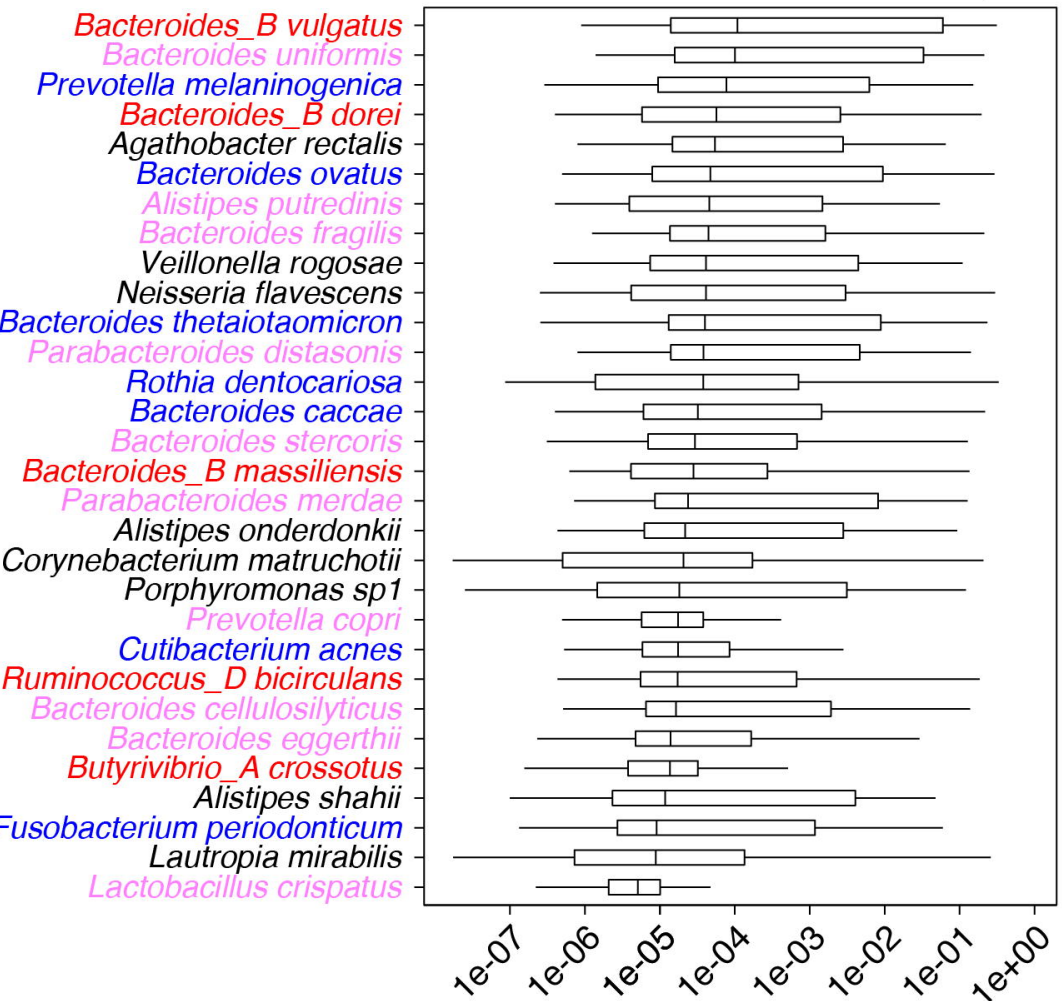

Species amongst top 30 most abundant species in:

One index only

NCBI_r88 and NCBI_r88 17k only (n=8)

All 3 indices $(n=7)$

Species definition unique to index 
Figure S7.

A

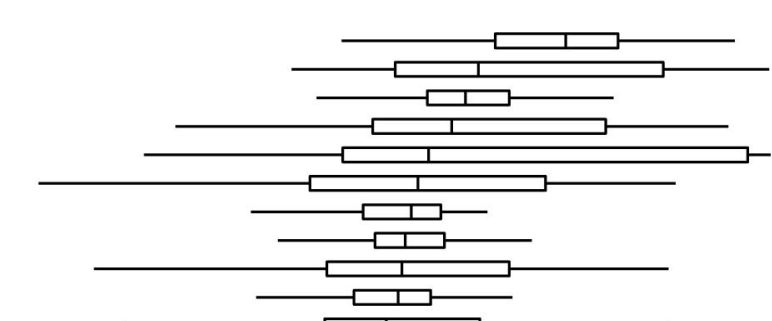

bioRxiv preprint doi: https://doi.org/10.1101/712166; this version posted July 23, the copyright holder for this pre

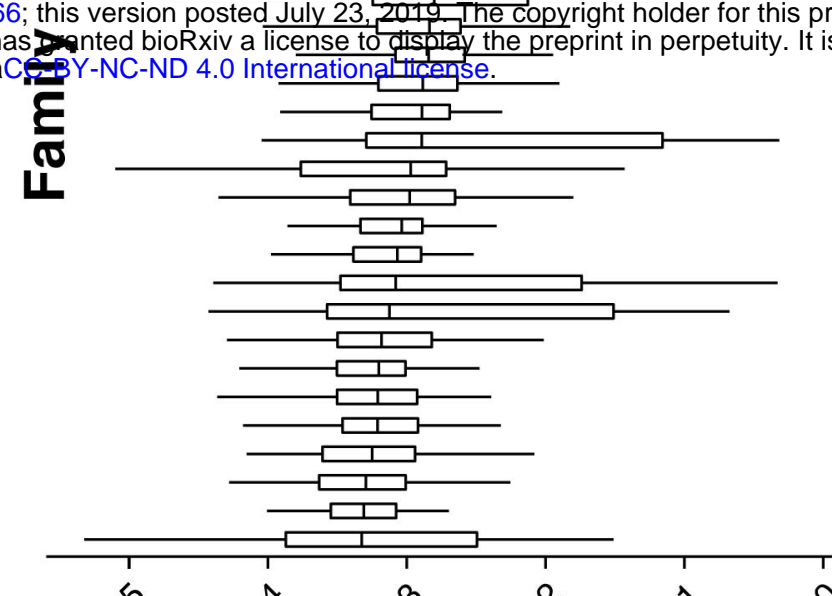

B

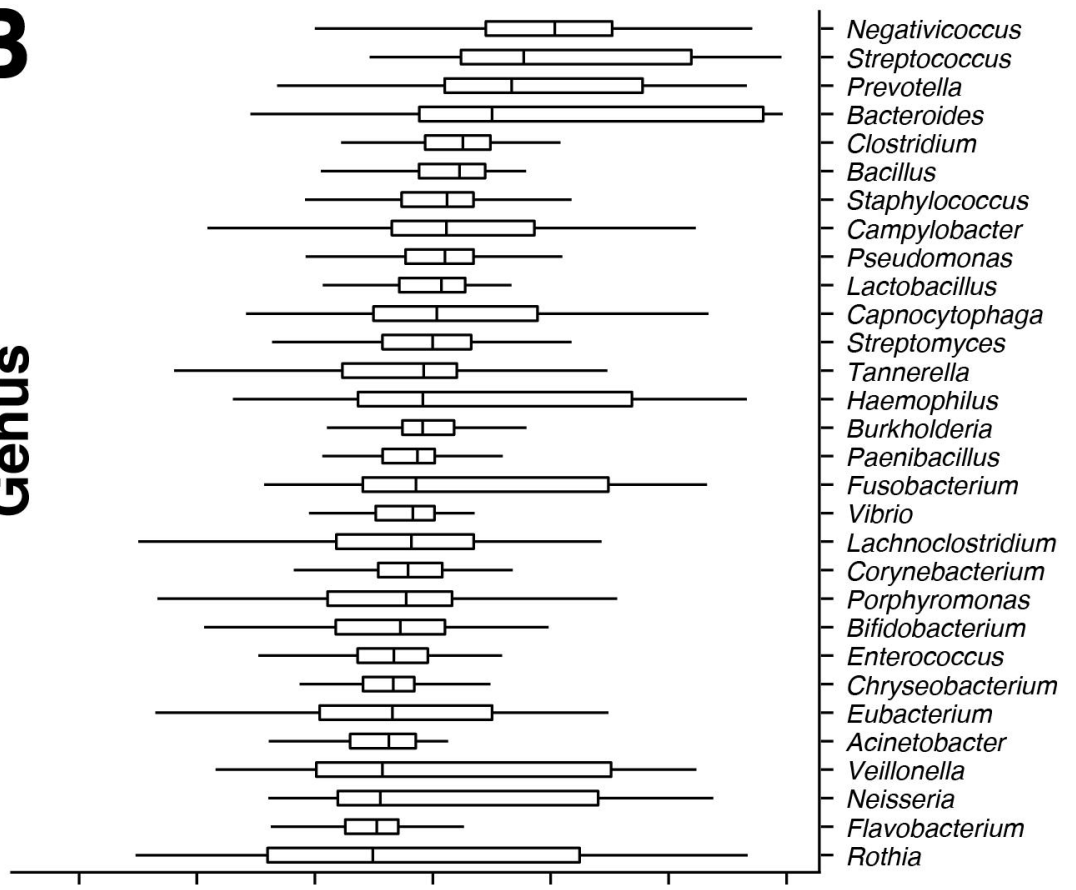

C

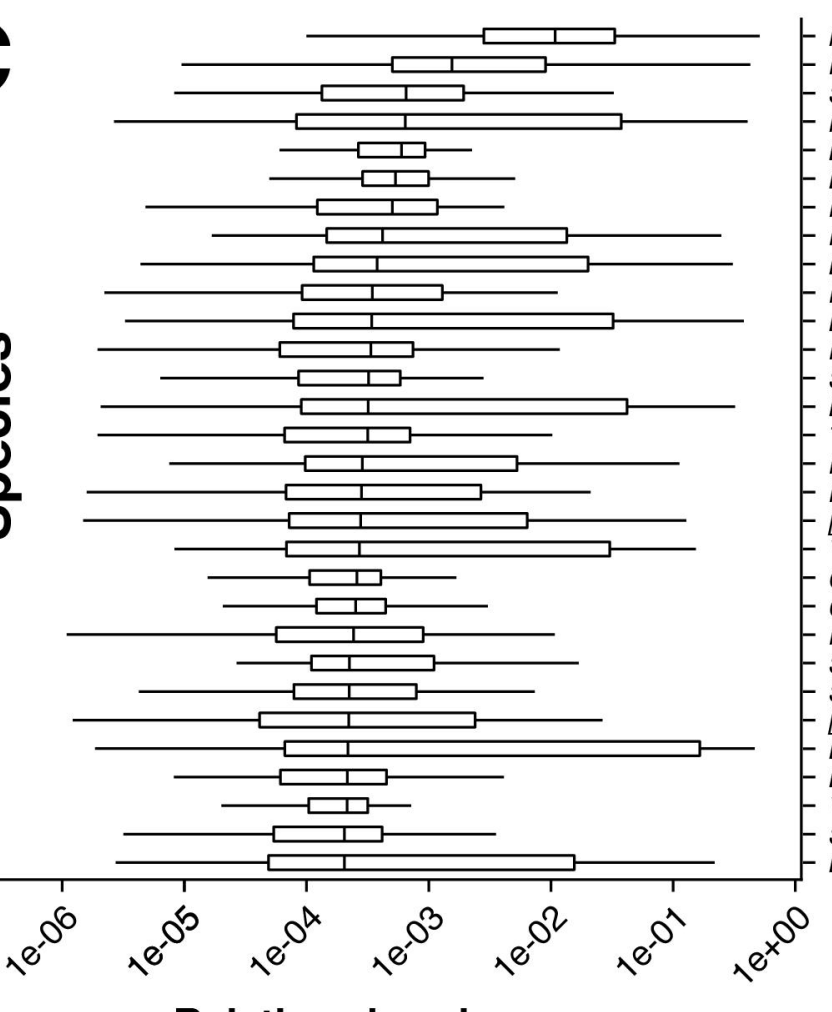

Taxon with exact correspondance in both NCBI and GTDB

Correspondance influenced by taxon redefinition / split in GTDB Taxon_A Taxon created in GTDB

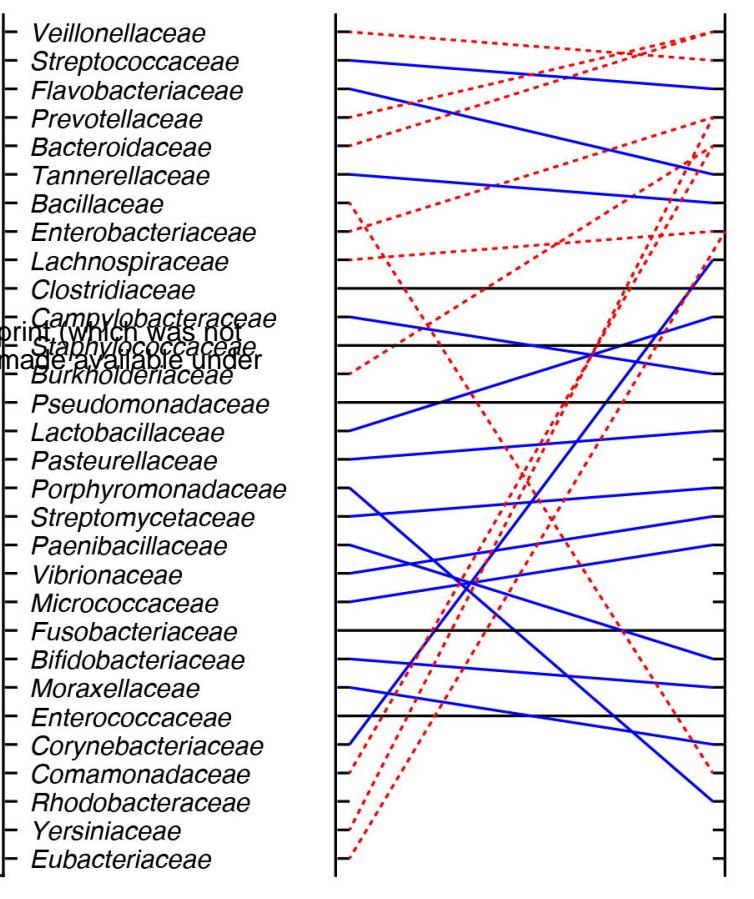

Bacteroidaceae
Negativicoccaceae
Streptococcaceae
Enterobacteriaceae
Burkholderiaceae
Flavobacteriaceae
Tannerellaceae
Lachnospiraceae
Corynebacteriaceae
Clostridiaceae
Lactobacillaceae
Staphylococcaceae
Campylobacteraceae
Pseudomonadaceae
Pasteurellaceae
Weeksellaceae
Streptomycetaceae
Vibrionaceae
Micrococcaceae
Rhizobiaceae
Altromonadaceae
Fusobacteriaceae
Paenibacillaceae
Bifidobacteriaceae
Enterococcacaceae
Moraxellaceae
Bacillaceae G
Porphyromonadaceae
Planococcaceae
Sphingomonadaceae

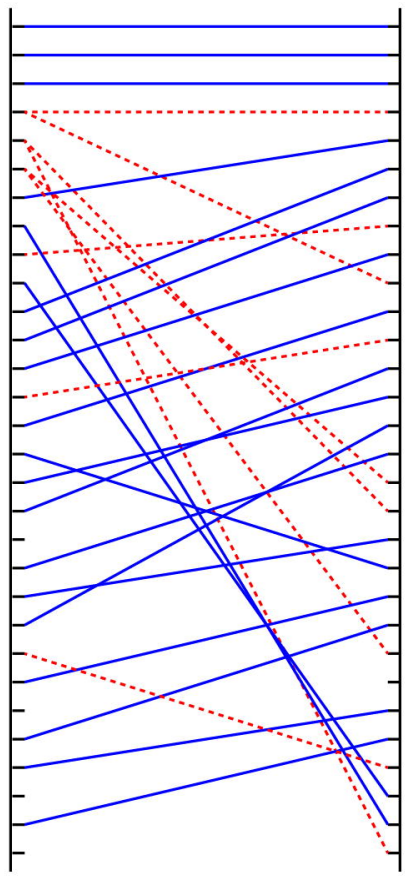

Negativicoccus

Negativicoccus
Streptococcus
Prevotella Prevotella
Bacteroides Staphylococcus Pseudomonas_E Bacteroides_ Burkholderia Haemophilus $D$ Fusobacterium Bifidobacterium
Corynebacterium
\begin{tabular}{l} 
Bacillus_A $A$ \\
Clostridium \\
\hline
\end{tabular} Porphyromonas
Paenibacillus Chryseobacterium
Acinetobacter Acinetobacter
Bacillus
Mycobacterium Flavobacterium Enterococcus $B$
Lactobacillus Campylobacter

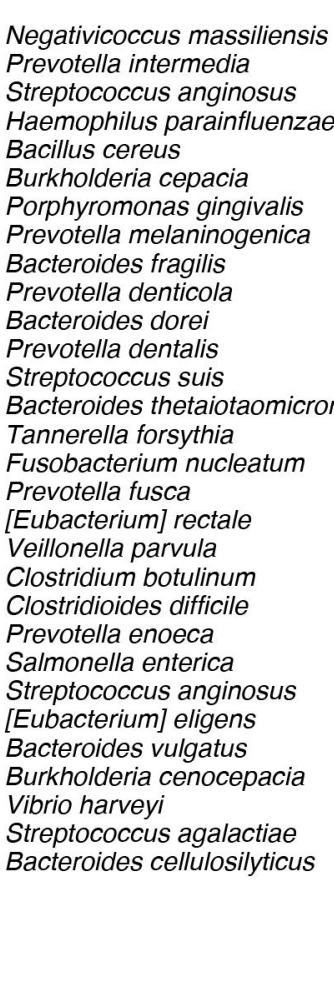

parainfluenz rkholderia cepacia tella melaninogenica

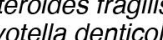
Frevotella fusca Veillonella parvula tella enoeca holderia cenocepacia (a) harvey teptococcus agalactiae

Relative abundance

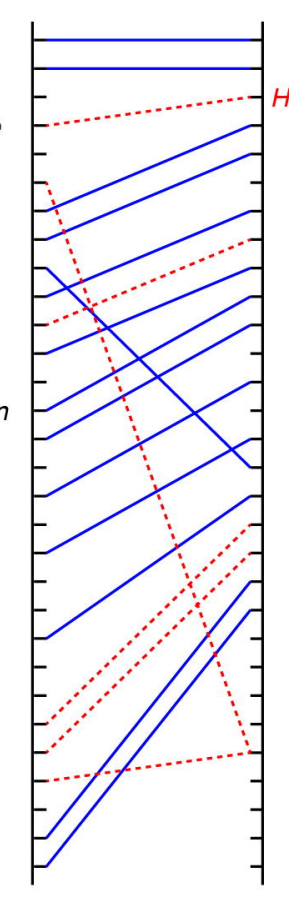

Negativicoccus massiliensis Porphyromonas gingivalis Prevotella melaninogenica Tannerella unknown $4571-$
Prevotella denticola Prevotella denticola
Bacteroides_B dorei Prevotella dentalis Bacteroides thetaiotaomicron Parabacteroides distasonis Prevotella fusca
A fustis
Vethobacter rectalis Veillonella parvula Bacteroides fragilis
Prevotella enoeca Lachnospira eligens_A Bacteroides_B vulgatus Streptococcus agalactiae
Bacteroides cellulosilyticus Odoribacter splanchnicus Bacteroides ovatus Faecalibacterium prausnitzii_C
Burkholderia cenocepacia_C Escherichia coli Streptococcus gordonii Streptococcus pneumoniae

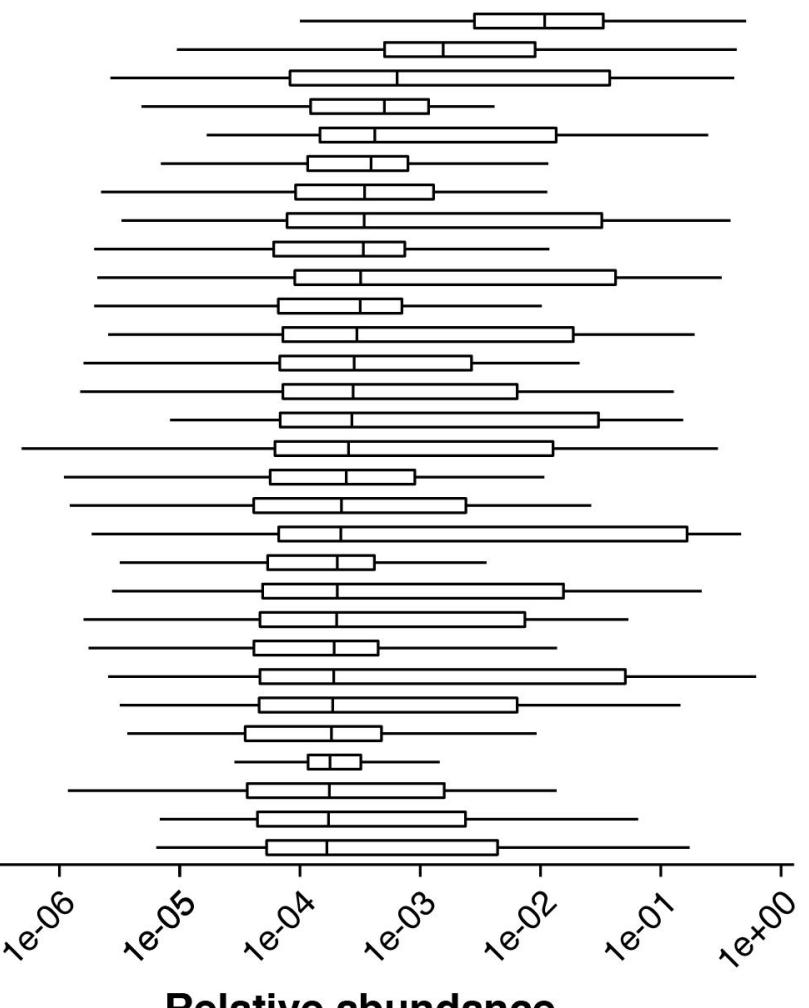

Relative abundance

GTDB_r86_8.6k
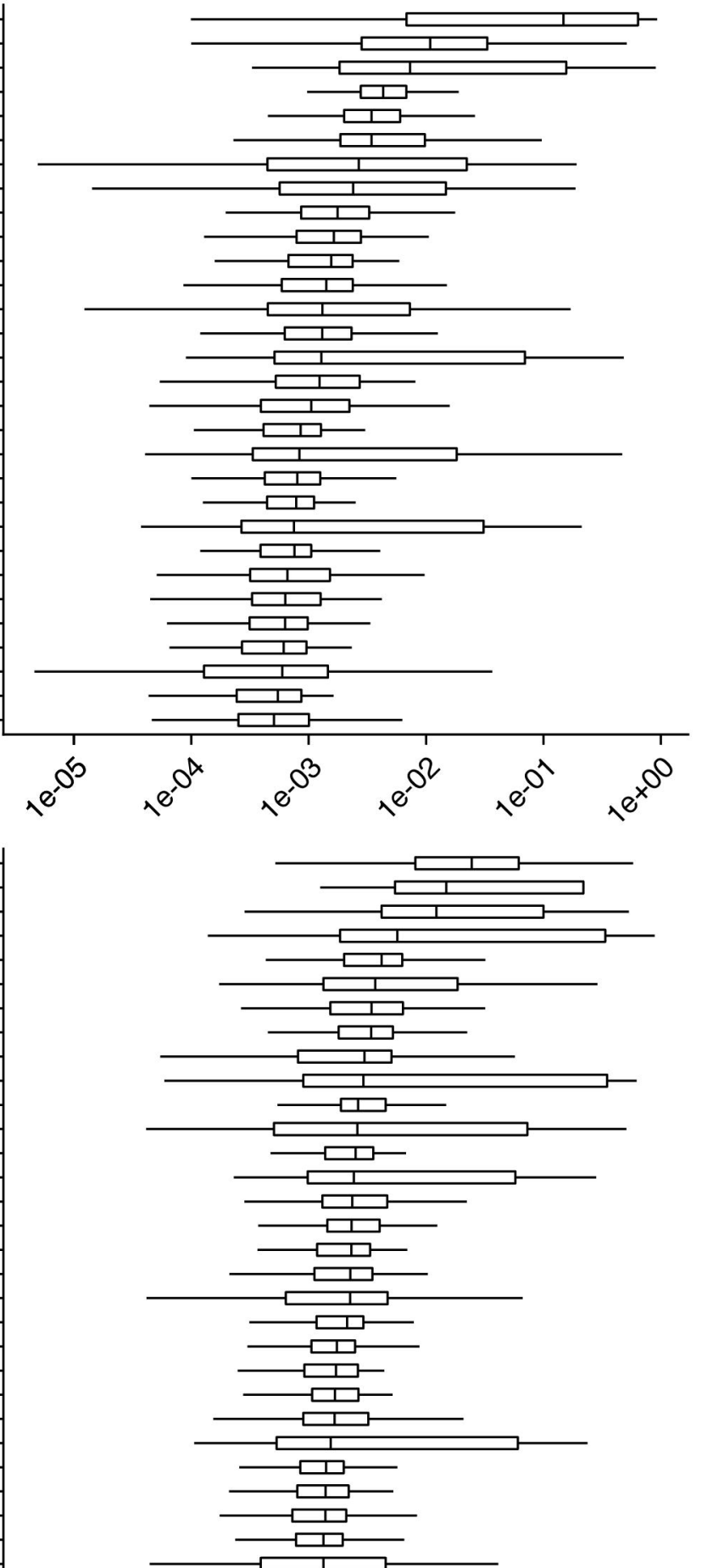

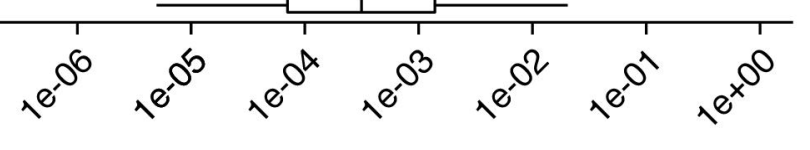




\section{Figure S8.}

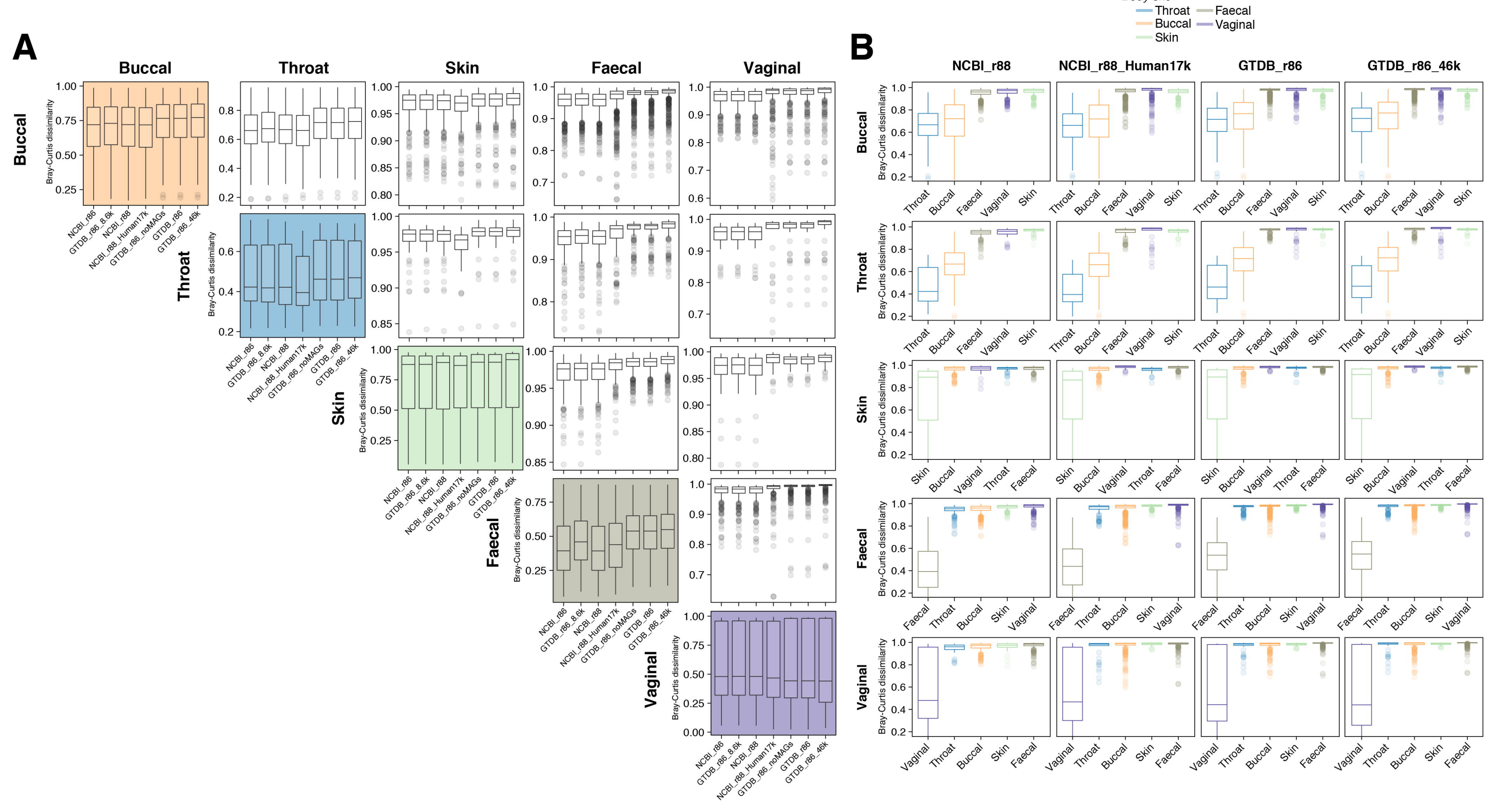


A

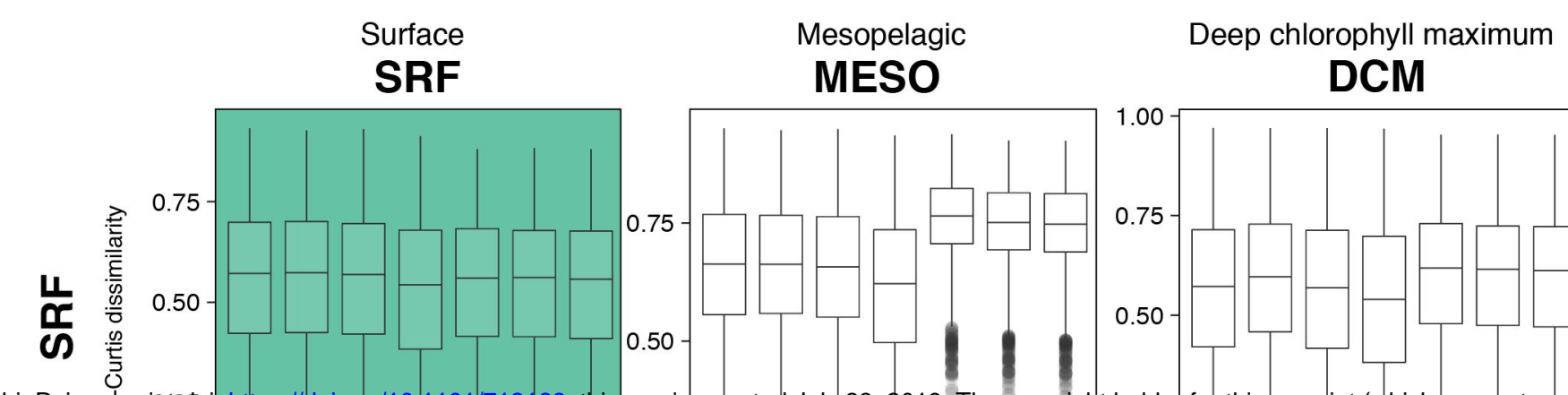

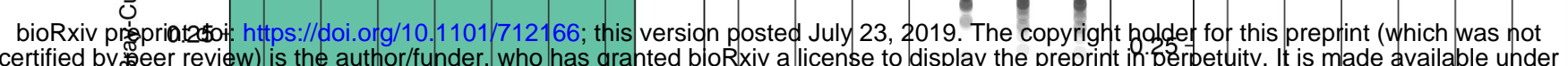
0.00
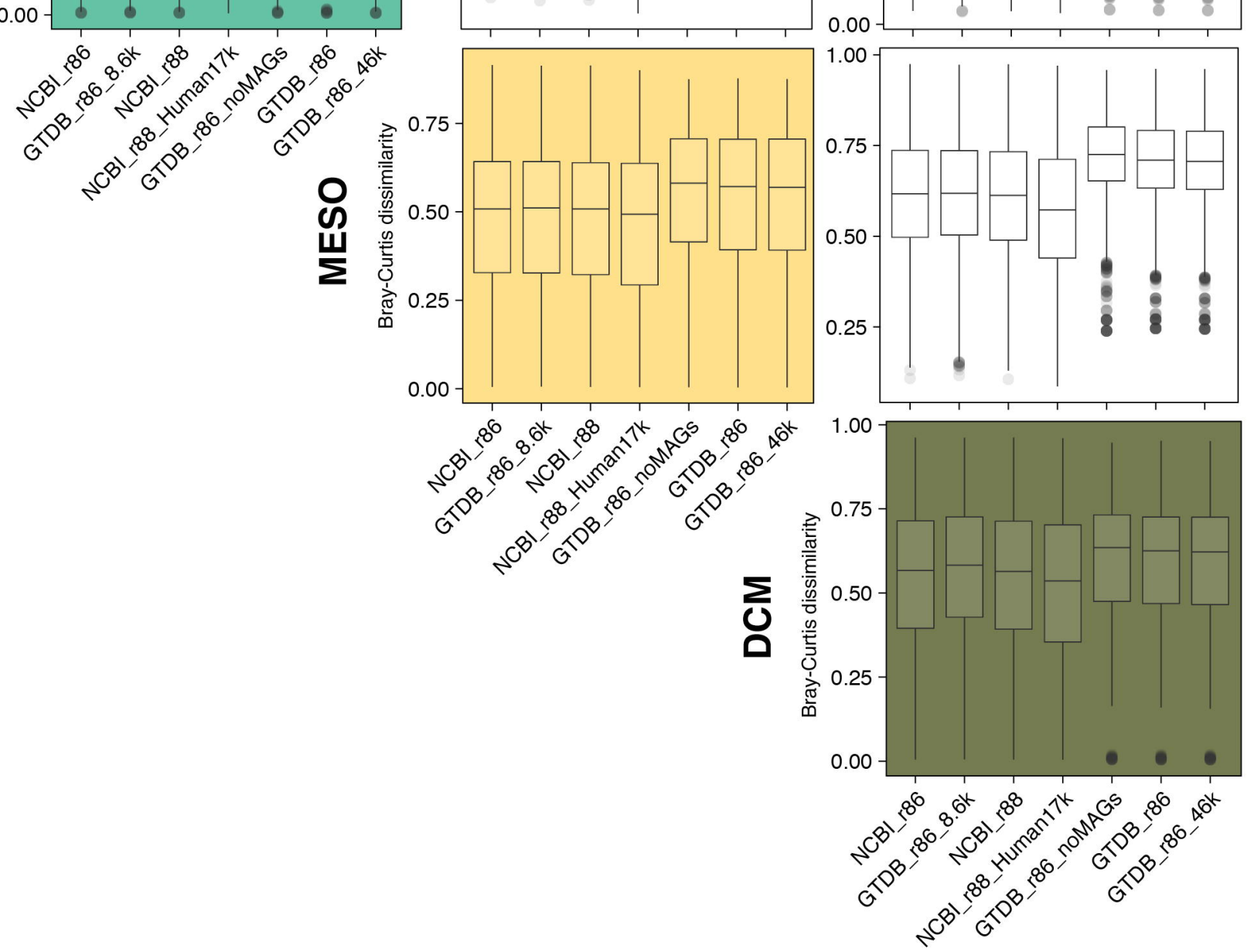

C

Cons

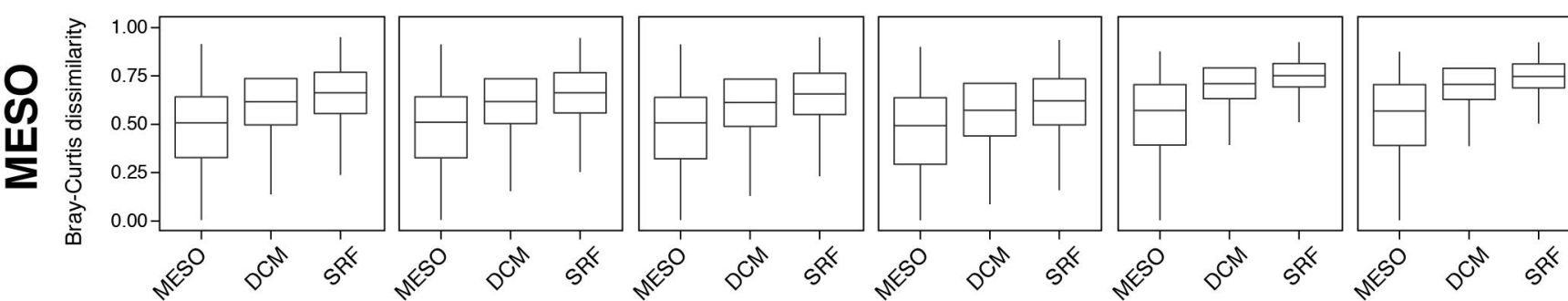

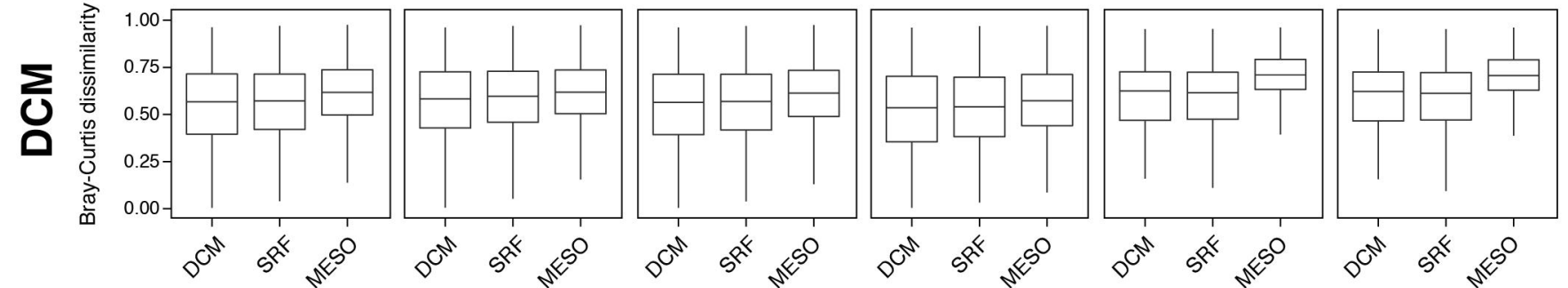

B

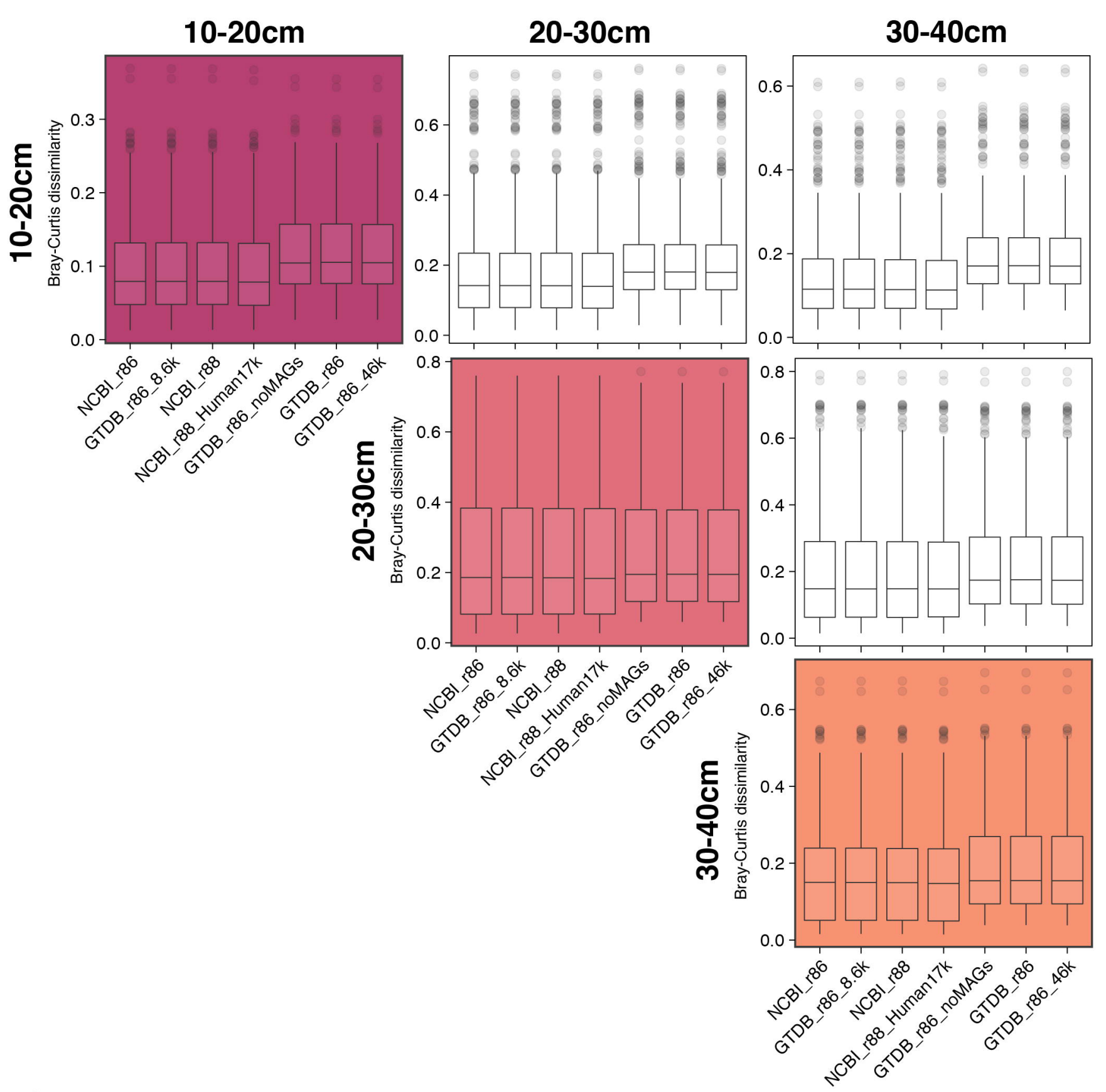

D

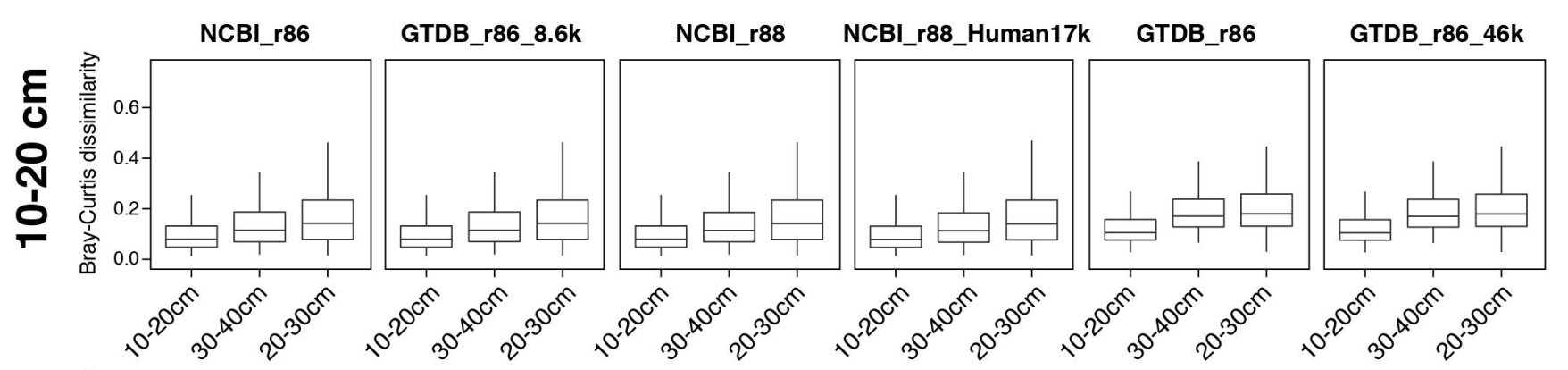

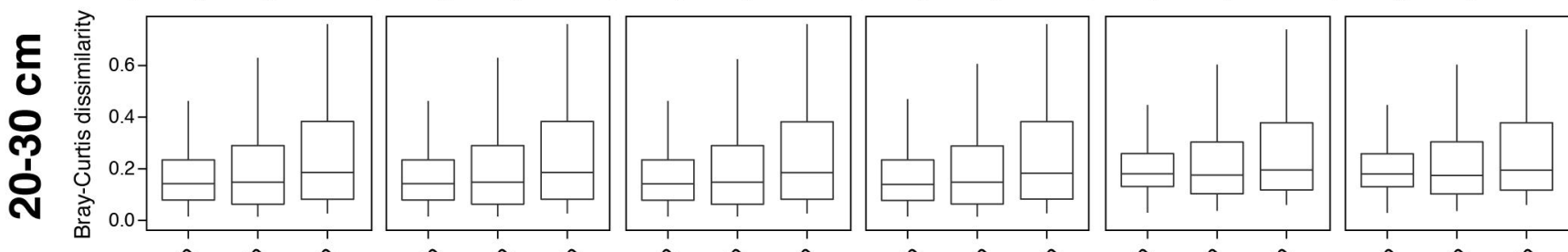

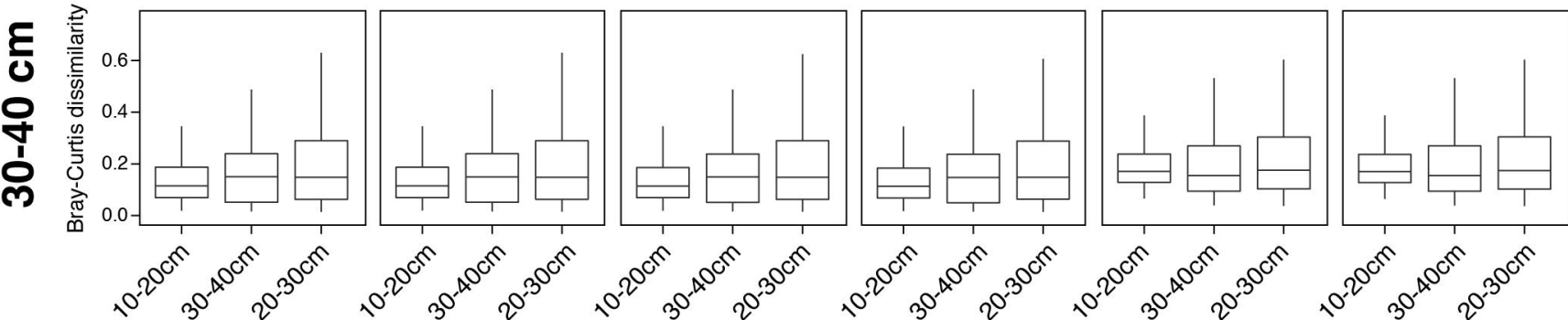




\section{Figure S10.}

HMP samples $(n=98)$
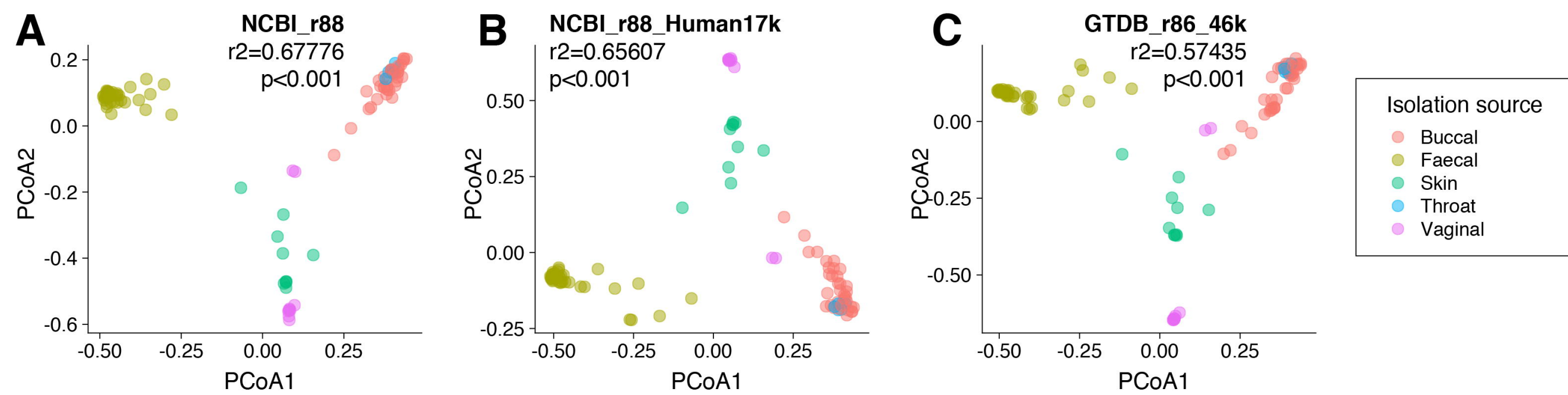

TARA Oceans samples $(n=246)$
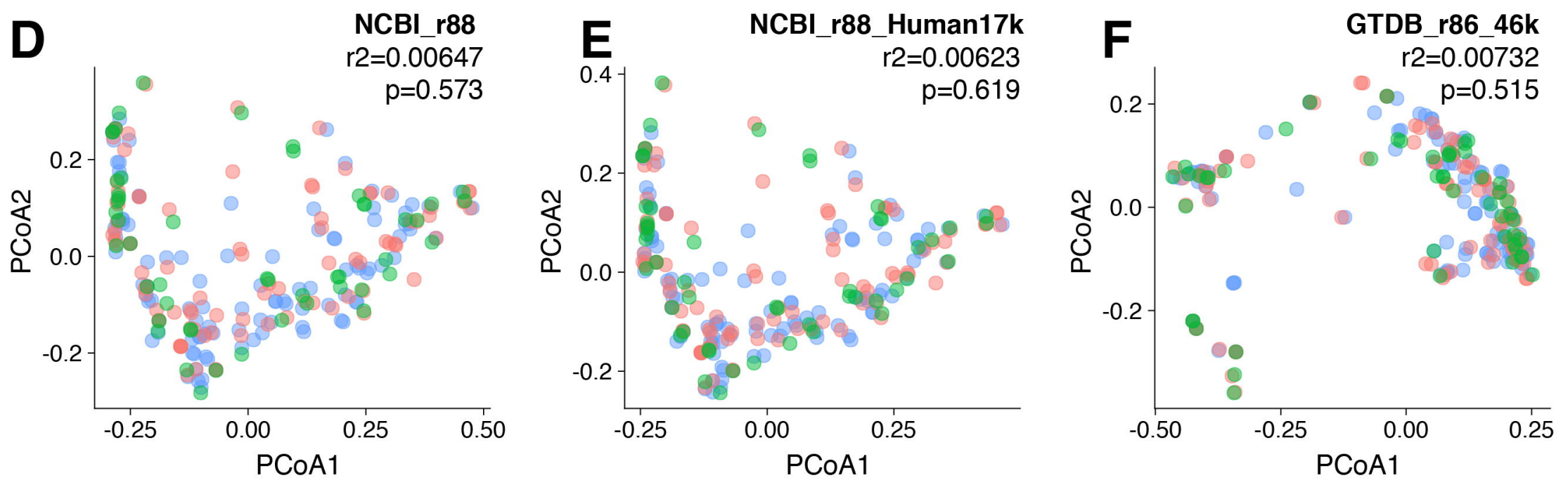

Depth layer group

- Deep Chlorophyll Maximum

Mesopelagic (MESO)

Surface (SRF)

Meadow soil samples $(n=60)$
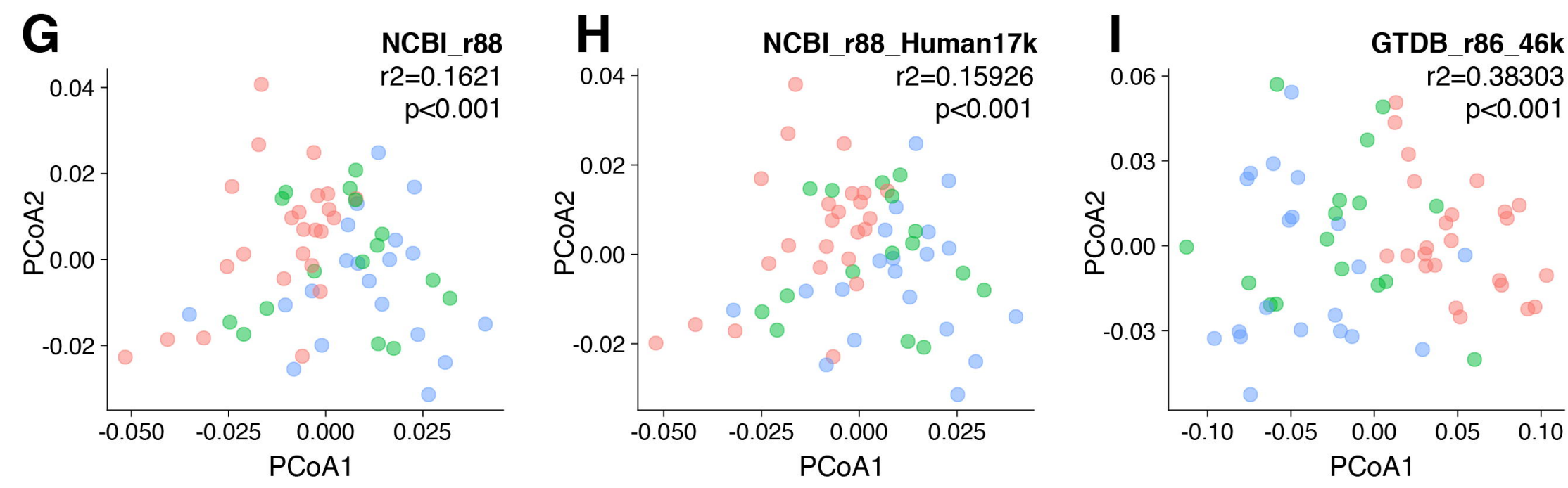

Sampling depth

- $10-20 \mathrm{~cm}$

$20-30 \mathrm{~cm}$

$30-40 \mathrm{~cm}$ 